\title{
Theoretical analysis of a fuzzy-logic controller with unequally spaced triangular membership functions ${ }^{1}$
}

\author{
Cheng-Liang Chen*, Sheng-Nan Wang, Chung-Tyan Hsieh, Feng-Yuan Chang \\ Department of Chemical Engineering, National Taiwan University, Taipei 10617, Taiwan, ROC
}

Received May 1996; received in revised form January 1997

\begin{abstract}
The mathematical analysis concerning the explicit input/output relation of the fuzzy logic controller (FLC) is addressed in this article. Under the assumption of simple rule mapping and the use of arbitrarily located triangular fuzzy partitions as the membership functions, the inferred output of the FLC can be decomposed into two terms: the global multilevel relay and the local nonlinear compensator. The ultimate control behavior of this FLC and the equivalence to nonlinear proportional-integral (PI) controller, as the number of linguistic terms are approaching to infinity, are also investigated. The local stability criteria for PI-type FLCs are derived and the global stability properties for PD-type FLCs are established. This analysis is a generalization to previous related research works where the FLC is characterized by equally spaced triangular fuzzy partitions on the universal sets. (C) 1999 Elsevier Science B.V. All rights reserved.
\end{abstract}

Keywords: Membership functions; Control theory; Analysis

\section{Introduction}

It has been two decades since the first paper on fuzzy control was published in 1974 [8]. A lot of fuzzy logic controllers (FLCs) in different areas have been reported in the literature and the fuzzy techniques have been widely used in home appliances.

However, one of the fundamental problems in fuzzy control technology is that there lacks a rigorous design theory on FLCs [18]. Until recently, many fuzzy logic controllers are constructed, instead of systematically designed, case by case by using the trial-and-error method guided by field operators' experiences on process operation. Such a condition sometimes leads to the FLC a black box that performs only the input/output mapping.

In order to make the behavior of the FLC more clear, Siler and Ying [10] propose a method to analyze the input/output parametric relationship of the simplest possible FLC. Since then, Ying [19,15-17] and some other authors such as [3] extend the analysis from the simplest FLC to the more complex FLCs and some useful results have been obtained.

${ }^{*}$ Corresponding author.

${ }^{1}$ Research supported by National Science Council, project number NSC86-2214-E-002-019. 
However, the membership functions used in the series of the above-mentioned analyses are all of equally spaced triangular/trapezoidal fuzzy partitions (ESMFs for short). It has been shown that the performance of such FLCs with ESMFs (designated as ESMFs-FLC) is on the same level as that of the linear PI controllers [3].

Recently, the authors propose a systematic design method of the FLCs [2]. This method uses a factor called shrinking factor to construct the membership functions called shrinking-span membership functions (SSMFs) of the linguistic variable. By only altering the shrinking factor, various unevenly spaced membership functions can be acquired and various nonlinear control actions could be obtained to cope with different control processes, either linear or nonlinear. Satisfactory simulation results of the FLC with SSMFs (SSMFs-FLC) for a wide variety of linear and nonlinear processes can be found in [2].

In this article, the authors like to extend the SSMFs-FLC to a more general case where the series of triangular fuzzy partitions could be arbitrarily distributed in an unequally spaced manner (USMFs-FLC for short) over the universal set, and the inner characteristics of the USMFs-FLC would be analyzed to elucidate the explicit input/output relation of this controller. Notably, the SSMFs-FLC and the ESMFs-FLC are two simplified varieties of the USMFs-FLC. Thus, this analysis is a generalization of previous related works $[19,15-17,3]$.

This paper is organized as follows: the basic structure of the FLC and the definition of USMFs (including SSMFs and ESMFs) are briefly reviewed in Section 2. Section 3 states the main results which include the input/output parametric relationship of the USMFs-FLC, the connection of the USMFs-FLC to the three mode (Proportional-Integral-Derivative, PID) controller, and the stability criteria of PI-type and PD-type FLCs, and finally, Section 4 concludes the main results.

\section{Structure of the USMFs-FLC}

As noted in [7], a fuzzy logic controller (FLC) comprises four principal components: a fuzzification interface (FI), a knowledge base (KB), decision-making logic (DML), and a defuzzification interface (DFI). Some complete and thorough description of the design procedures of the FLC have been presented (see $[4,13]$ ); therefore, only the essential material will be reviewed here as a fundamental work. Readers should refer to the literatures listed above for details.

\subsection{Components of the USMFS-FLC}

In most of the applications of the FLC, the input linguistic variables are usually error $(e)$ and change in error $(r)$ of the process output, and the output linguistic variable is the change in controller output $(u)$. Without loss of generality, it is assumed that the inputs $(e, r)$ have been scaled from the measurements and the output $(u)$ would be scaled before being applied to the processes. Thus, the associative universe of discourse of $e, r, u$ are confined within \pm 1 , i.e., $U_{e}=U_{r}=U_{u}=[-1,1]$. Furthermore, each of the three term-sets $\left(T_{e}, T_{r}, T_{u}\right)$ individually has the same number of linguistic members on both positive/negative sides:

$$
\begin{aligned}
& T_{e}=\left\{E_{-m_{e}}, \ldots, E_{-1}, E_{0}, E_{1}, \ldots, E_{m_{e}}\right\}=\left\{E_{i} \mid i \in I_{m_{e}}\right\}, \\
& T_{r}=\left\{R_{-m_{r}}, \ldots, R_{-1}, R_{0}, R_{1}, \ldots, R_{m_{r}}\right\}=\left\{R_{j} \mid j \in I_{m_{r}}\right\}, \\
& T_{u}=\left\{U_{-m_{u}}, \ldots, U_{-1}, U_{0}, U_{1}, \ldots, U_{m_{u}}\right\}=\left\{U_{k} \mid k \in I_{m_{u}}\right\} .
\end{aligned}
$$

Here $I_{m_{e}}=\left\{-m_{e}, \ldots,-1,0,1, \ldots, m_{e}\right\}$ is the index set with $M_{e}=2 m_{e}+1$ terms for linguistic variable $e$. That is, the universe of discourse $U_{e}$ of linguistic variable $e$ is partitioned into $M_{e}$ sections and each section is associated with a linguistic term $E_{i}, i \in I_{m_{i}}$, and is characterized by the membership function $E_{i}(e) . m_{r}, m_{u}$, $M_{r}, M_{u}, I_{m_{r}}$, and $I_{m_{u}}$ are defined similarly. 
In this article, the simple control rule mapping [12] in the rule base is used:

$$
R_{i, j}: \quad \text { IF } e \text { is } E_{i} \text { AND } r \text { is } R_{j} \text { THEN } u \text { is } U_{k=i+j} \text {. }
$$

The simple rule base implies $m_{u}=m_{e}+m_{r}$. In order to make the FLC easier to implement, it can be further assumed that $T_{e}$ and $T_{r}$ have the same number of elements, that is, $m_{e}=m_{r}=m$ and therefore $m_{u}=m_{e}+$ $m_{r}=2 m$. Notably, this assumption results in a bi-symmetric FLC, i.e., the output of rule $R_{i, j}$ equals to the output of $R_{u, v}$ if $i+j=u+v$. This is, of course, not a natural property of most FLCs in practice. However, such assumption is rational for initial design and we adopt it to simplify the subsequent mathematical analysis.

This two-input-one-output fuzzy feedback control system and its detailed computational steps are depicted as follows:

$$
\begin{aligned}
& e_{m}(n T)=\left.\{s p(t)-y(t)\}\right|_{t=n T} \in\left[-\frac{1}{\mathrm{GE}}, \frac{1}{\mathrm{GE}}\right], \\
& r_{m}(n T)=e_{m}(n T)-e_{m}(n T-T) \in\left[-\frac{1}{\mathrm{GR}}, \frac{1}{\mathrm{GR}}\right], \\
& e^{*}=\mathrm{GE} e_{m}(n) \in[-1,1], \quad r^{*}=\mathrm{GR} r_{m}(n) \in[-1,1], \\
& E_{i}\left(e^{*}\right)=\mathbb{M}\left(e^{*}, E_{i}\right) \in[0,1], i \in I_{m_{e}}, \quad R_{j}\left(r^{*}\right)=\mathbb{M}\left(r^{*}, R_{j}\right) \in[0,1], j \in I_{m_{i}}, \\
& \phi_{i, j}=\mathbb{E}\left(E_{i}\left(e^{*}\right), R_{j}\left(r^{*}\right)\right) \in[0,1], \\
& U_{i+j}^{\prime}(u)=\mathbb{U}\left(\phi_{i, j}, U_{i+j}(u)\right), \quad U(u)=\underset{\forall i, j \in I_{m}}{\mathbb{A}} U_{i+j}^{\prime}(u), \\
& u^{*}=\mathbb{D}(U(u)) \in[-1,1], \quad u_{m}(n T)=\mathrm{GU} u^{*} \in[-\mathrm{GU}, \mathrm{GU}], \\
& \operatorname{CO}(n T)=\mathrm{CO}(n T-T)+u_{m}(n T) \quad \text { for PI-type FLC, } \\
& \operatorname{CO}(t)=\mathrm{CO}(n T) \quad \text { for } t \in[n T, n T+T] .
\end{aligned}
$$

Here $T$ is the sampling interval; $s p(t), \mathrm{CO}(t)$, and $y(t)$ denote set-point, process input, and process output, respectively; $e_{m}(n T), r_{m}(n T)$, and $u_{m}(n T)\left(e_{m}, r_{m}, u_{m}\right.$ for short $)$ are measured error, change in error, and final crisp output without scaling, respectively; GE, GR, and GU are scalars, which keep the scaled crisp values $e^{*}, r^{*}$, and $u^{*}$ within numerical range of $[-1,1] ; E_{i}\left(e^{*}\right)$ and $R_{j}\left(r^{*}\right)$ are the membership grade for $e$ and $r$,

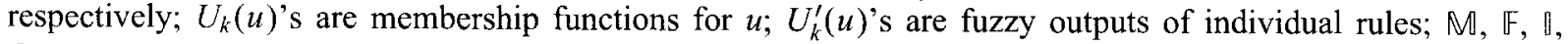
$\mathbb{A}$, and $\mathbb{D}$ are a series of operators performing the decision making in the FLC with their specific functions [2]: Operator $\mathbb{M}$ maps a crisp input value to a membership degree subjected to a specific membership function; $\mathbb{F}$ is used to calculate the firing level of each of the rules in the rule base; d decides the output fuzzy set of each rule; $\mathbb{A}$ aggregates the individual fuzzy outputs to form the overall output fuzzy set $U(u)$; finally, $\mathbb{D}$ converts the fuzzy output $U(u)$ into a single crisp value $u^{*}$ for implemention.

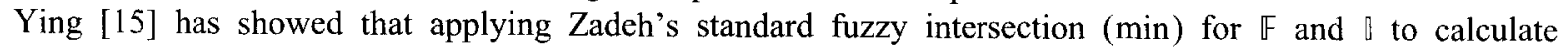
the firing level $\phi_{i, j}$ and individual fuzzy output $U_{i+j}^{\prime}(u)$, using a crisp value $\bar{u}_{i+j}$ in place of the fuzzy output set, employing Lukasiewicz's fuzzy union for $\mathbb{A}$ to get the combined output membership function, and finally the center-of-gravity method for $\mathbb{D}$, then the final crisp output can be obtained by the following equation:

$$
u_{m}=\mathrm{GU} \times u^{*}=\mathrm{GU} \times \frac{\sum_{\phi_{i, j} \neq 0} \phi_{i, j} \bar{u}_{i+j}}{\sum_{\phi_{i, j} \neq 0} \phi_{i, j}} .
$$


Thus, the remaining problem in obtaining the explicit input/output relation, $u_{m}=f\left(e^{*}, r^{*}\right)$, is to find out the firing level of each rule, and to assign a crisp output representative, $\bar{u}_{i+j}$, for each rule for given input pattern $\left(e^{*}, r^{*}\right)$ within the universal domain $U_{e} \times U_{r}$. In this article, we use the centroid of a membership function as its representative crisp value. As for the membership functions, three kinds of triangular fuzzy partitions will be discussed in the next subsection.

\subsection{Unequally spaced triangular membership functions (USMFs)}

In the real applications of FLCs, the membership functions are usually constructed by assembling knowledge of the domain experts and then modified by laboriously surveying the control response of the process. In most of the control cases, the FLCs cannot be effective without carefully arranging the membership functions.

In the theoretical analysis of the FLC, however, the selection of membership functions does not get much attention in the majority of researches. Most researchers choose isosceles triangles with equal spans throughout the whole universe of discourse as membership functions for their FLCs $[8,10,19,12]$. The main advantage of choosing this type of membership functions is that equal span isosceles triangular membership functions ease the difficulties in analyzing the structure of the FLC. However, most practical applications of FLCs adopt nonequal-span membership functions to cope with the real control problems such as the water purification process [14], the truck backer-upper system [6], and the neutralization processes [1], etc. Instinctively, the closer the control response to the set point (or normal condition), the narrower ranges of membership functions should be to increase the sensitivity of the FLC to small input variations around set point (for example, see [4, p. 203; 6, p. 341]). For some highly nonlinear processes, such as the inverted pendulum system or the neutralization process, an FLC with equal span triangular membership functions is not adequate to achieve good control result [1].

In order to accomplish a better performance and to devise a more rational FLC, a successively distributed triangular fuzzy partitions would be used as the membership functions. Let $E_{i}^{*}$ 's, $i \in I_{m_{e}}$, be a series of principal values (cores) for $e$ which are successively distributed on $U_{e}$ with unequal spacings. Then the unequally spaced triangular membership functions (USMFs for short) for $e, E_{i}(e)$ 's, are defined such that $E_{i}(e)$ is in triangular shape with principal value at $E_{i}^{*}$, i.e., $E_{i}\left(e^{*}=E_{i}^{*}\right)=1$, and its support set is restricted within $\left[E_{i-1}^{*}, E_{i+1}^{*}\right] \in U_{e}$ for all $i \in I_{m_{e}}$ :

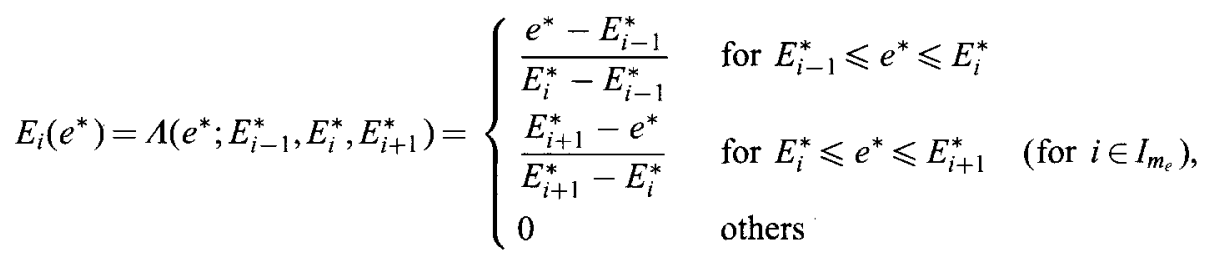

where $-1=E_{-m_{e}}^{*} 0<\cdots<E_{-1}^{*}<E_{0}^{*}<E_{1}^{*}<\cdots<E_{m_{e}}^{*}=1$.

In order to comply with the representation above, let $E_{-m_{e}-1}^{*}=E_{-m_{e}}^{*}=-1$ and $E_{m_{e}+1}^{*}=E_{m_{e}}^{*}=1$.

Notably when these $E_{i}^{*}$ 's are ordered in a more specific way such that $E_{i}^{*}=\left(i / m_{e}\right) s_{e}^{m_{e}-|i|}$, where $s_{e} \in(0,1]$ is the shrinking factor for linguistic variable $e$, the USMFs would be reduced into a series of shrinkingspan membership functions (SSMFs for short) [2]. The SSMFs could be further reduced into equally-spaced membership functions (ESMFs for short) if it has unity shrinking factor $\left(s_{e}=1\right.$ ), and in such case the spacing would become $\left(1 / m_{e}\right)$. Fig. 1 gives typical USMFs, SSMFs, and ESMFs for $e$. The definitions for $R_{j}(r)$ 's and $U_{k}(u)$ 's can be established in the same way.

An FLC equipped with USMFs (SSMFs, ESMFs) is named USMFs-FLC (SSMFs-FLC, ESMFs-FLC). Notably, the SSMFs-FLC with $s_{e}=s_{r}=s_{u}=1$ is equivalent to an ESMFs-FLC that has been analyzed thoroughly [15-17]. Hence, the following analysis is a generalization of those previous related works. 


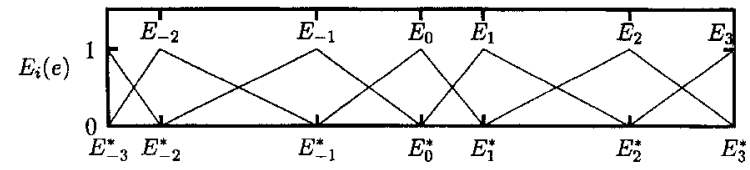

(a)

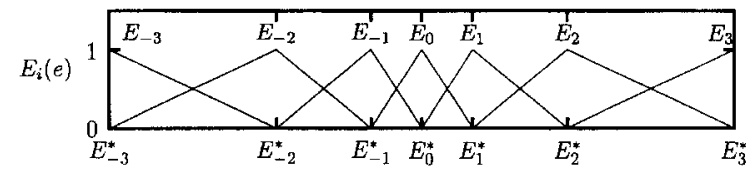

(b)

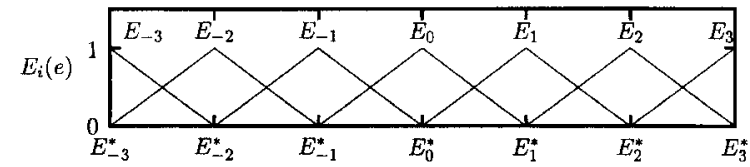

(c)

Fig. 1. Typical membership functions for $e(m=3)$ : (a) USMFs, (b) SSMFs $(s=0.7$ ), and (c) ESMFs.

\section{Theoretical analysis of the USMFs-FLC}

Some interesting properties of the USMFs and the USMFs-FLC will be studied in this section. The following discussion, except Section 3.5, would be focused on PI-type FLCs.

\subsection{Basic properties of USMFS}

The following two properties can be obtained straightforwardly [9] for using the triangular fuzzy partitions as the membership functions.

Property 1. Given numerical value $e^{*} \in\left[E_{i}^{*}, E_{i+1}^{*}\right] \subset U_{e}$ for $e$ and $r^{*} \in\left[R_{j}^{*}, R_{j+1}^{*}\right] \subset U_{r}$ for $r$ where $i \in I_{m_{l}}$ $-\left\{m_{e}\right\}$ and $j \in I_{m_{r}}-\left\{m_{r}\right\}$, there exist only two non-zero membership values in the USMFs for each of the two input variables, that is,

$$
E_{\hat{i}}\left(e^{*} \in\left[E_{i}^{*}, E_{i+1}^{*}\right]\right) \begin{cases}\neq 0 & \text { if } \hat{i}=i, i+1, \\ =0 & \text { otherwise }\end{cases}
$$

and

$$
R_{\hat{j}}\left(r^{*} \in\left[R_{j}^{*}, R_{j+1}^{*}\right]\right) \begin{cases}\neq 0 & \text { if } \hat{j}=j, j+1, \\ =0 & \text { otherwise. }\end{cases}
$$

Due to this property, it is not necessary to calculate the membership values for all USMFs and this greatly reduces the computational effort in implementing USMFs in real industrial processes.

Property 2. The two non-zero membership values in the USMFs of Property 1 have a sum of 1 and hence the summation of all membership values for $e$ (and $r$ ) is also 1. That is,

$$
\sum_{i \in I_{m_{t^{*}}}} E_{i}\left(e^{*}\right)=1 \quad \forall e^{*} \in U_{e}, \quad \sum_{j \in I_{m_{*}}} R_{j}\left(r^{*}\right)=1 \quad \forall r^{*} \in U_{r}
$$


The centroids of the individual membership functions of output variable $u$ are required in calculating the final USMFs-FLC output. Property 3 states that the centroid of a single membership function (local centroid) with the form (5) can be computed by using the principal values only.

Property 3. For the USMFs $U_{k}(u)$ 's defined as

$$
U_{k}(u)=\Lambda\left(u ; U_{k-1}^{*}, U_{k}^{*}, U_{k+1}^{*}\right) \text { for } k \in I_{m_{u}},
$$

let $U_{-m_{u}-1}^{*}=U_{-m_{u}}^{*}=-1$ and $U_{m_{u}+1}^{*}=U_{m_{u}}^{*}=1$, and let $\bar{u}_{k}$ 's be the centroids of membership functions $U_{k}(u)$ 's. Then

$$
\bar{u}_{k}=\left(\frac{U_{k-1}^{*}+U_{k}^{*}+U_{k+1}^{*}}{3}\right) \quad \text { for } k \in I_{m_{u}} .
$$

Proof. In computing the centroid value for membership function $U_{k}(u)$, the widely used center of area (COA) method is adopted here to generate the result. In the case of continuous universe of discourse, this method gives

$$
\bar{u}_{k}=\frac{\int_{U_{u}} U_{k}(u) u \mathrm{~d} u}{\int_{U_{u}} U_{k}(u) \mathrm{d} u} .
$$

The numerator of Eq. (7) is referred as moment and the denominator is called area of the membership function for convenience.

Then, the moment, $M_{k}$, of the membership function $U_{k}(u)$ is

$$
\begin{aligned}
M_{k} & =\int_{U_{u}} U_{k}(u) u \mathrm{~d} u=\int_{U_{k-1}^{*}}^{U_{k}^{*}}\left(\frac{u-U_{k-1}^{*}}{U_{k}^{*}-U_{k-1}^{*}}\right) u \mathrm{~d} u+\int_{U_{k}^{*}}^{U_{k+1}^{*}}\left(\frac{U_{k+1}^{*}-u}{U_{k+1}^{*}-U_{k}^{*}}\right) u \mathrm{~d} u \\
& =\frac{1}{6}\left(U_{k+1}^{*}-U_{k-1}^{*}\right)\left(U_{k-1}^{*}+U_{k}^{*}+U_{k+1}^{*}\right) .
\end{aligned}
$$

The area, $S_{k}$, is

$$
S_{k}=\int_{U_{v}} U_{k}(u) \mathrm{d} u=\int_{U_{k-1}^{*}}^{U_{k}^{*}}\left(\frac{u-U_{k-1}^{*}}{U_{k}^{*}-U_{k-1}^{*}}\right) \mathrm{d} u+\int_{U_{k}^{*}}^{U_{k+1}^{*}}\left(\frac{U_{k+1}^{*}-u}{U_{k+1}^{*}-U_{k}^{*}}\right) \mathrm{d} u=\frac{1}{2}\left(U_{k+1}^{*}-U_{k-1}^{*}\right) .
$$

Therefore, the centroid of membership function $U_{k}(u)$ is

$$
\bar{u}_{k}=\frac{M_{k}}{S_{k}}=\frac{1}{3}\left(U_{k-1}^{*}+U_{k}^{*}+U_{k+1}^{*}\right) .
$$

This completes the proof.

\subsection{Input-output relation of the USMFs-FLC}

For the USMFs-FLCs depicted in Section 2.1, it is obvious from Properties 1 and 2 that only four control rules have nonzero firing levels for specific input values $\left(e^{*}, r^{*}\right)$.

Property 4. Given numerical value $e^{*} \in\left[E_{i}^{*}, E_{i+1}^{*}\right] \subset U_{e}$ for $e$ and $r^{*} \in\left[R_{j}^{*}, R_{j+1}^{*}\right] \subset U_{r}$ for $r$, then only the following four control rules have nonzero output (where $k=i+j ; i, j \in I_{m}-\{m\}$ ):

(r1) if $e$ is $E_{i+1}$ and $r$ is $R_{j+1}$ then $u$ is $U_{k+2}$,

(r2) if $e$ is $E_{i+1}$ and $r$ is $R_{j}$ then $u$ is $U_{k+1}$,

(r3) if $e$ is $E_{i}$ and $r$ is $R_{j+1}$ then $u$ is $U_{k+1}$,

(r4) if $e$ is $E_{i}$ and $r$ is $R_{j}$ then $u$ is $U_{k}$. 


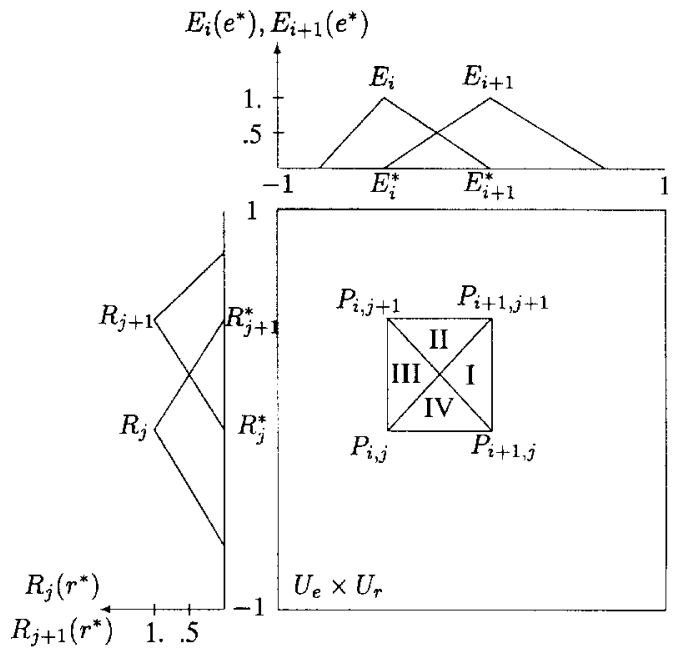

Fig. 2. The $i j$ th-block for $e$ and $r$ on the domain $U_{e} \times U_{r}$.

For the various combinations of crisp input values $e^{*}$ and $r^{*}$ of the USMFs-FLC, the $i j$ th-block is used to refer the rectangular region in which $e^{*} \in\left[E_{i}^{*}, E_{i+1}^{*}\right] \subset U_{e}$ and $r^{*} \in\left[R_{j}^{*}, R_{j+1}^{*}\right] \subset U_{r}$. Points $P_{i, j}=\left(E_{i}^{*}, R_{j}^{*}\right)$, $P_{i+1, j}=\left(E_{i+1}^{*}, R_{j}^{*}\right), P_{i+1, j+1}=\left(E_{i+1}^{*}, R_{j+1}^{*}\right)$, and $P_{i, j+1}=\left(E_{i}^{*}, R_{j+1}^{*}\right)$ are used to denote the four corners of the $i j$ th-block as shown in Fig. 2. All the points locate on the line $\overline{P_{i, j} P_{i+1, j+1}}$ have the same values for $E_{i}\left(e^{*}\right)$ and $R_{j}\left(r^{*}\right)$; the points below the line $\overline{P_{i, j} P_{i+1, j+1}}$ have the characteristic that $E_{i}\left(e^{*}\right)<R_{j}\left(r^{*}\right)$, and for those above the line $\overline{P_{i, j} P_{i+1, j+1}}, E_{i}\left(e^{*}\right)>R_{j}\left(r^{*}\right)$. Similar relations can be obtained for the line $\overline{P_{i+1, j} P_{i, j+1}}$ : for the points below the line, $E_{i}\left(e^{*}\right)>R_{j+1}\left(r^{*}\right)$; for those above the line, $E_{i}\left(e^{*}\right)<R_{j+1}\left(r^{*}\right)$; and for those on the line, $E_{i}\left(e^{*}\right)=R_{j+1}\left(r^{*}\right)$. Note that $E_{i}\left(e^{*}\right) \leqslant(\geqslant) R_{j}\left(r^{*}\right)$ is equivalent to $E_{i+1}\left(e^{*}\right) \geqslant(\leqslant) R_{j+1}\left(r^{*}\right)$ and $E_{i}\left(e^{*}\right) \leqslant(\geqslant) R_{j+1}\left(r^{*}\right)$ is the same as $E_{i+1}\left(e^{*}\right) \geqslant(\leqslant) R_{j}\left(r^{*}\right)$.

According to the relative magnitude of the membership values of $E_{i}\left(e^{*}\right), E_{i+1}\left(e^{*}\right), R_{j}\left(r^{*}\right)$, and $R_{j+1}\left(r^{*}\right)$, four regions are partitioned to represent the four different combinations of the membership values within the $i j$ th-block such as stated in the following property and shown in Fig. 2.

Property 5. For a specific ijth-block in the domain of $U_{e} \times U_{r}$, four regions are partitioned with the following relations:

- Region I: $E_{i}\left(e^{*}\right) \leqslant R_{j}\left(r^{*}\right)$ and $E_{i}\left(e^{*}\right) \leqslant R_{j+1}\left(r^{*}\right)\left(\right.$ or $\left.E_{i}\left(e^{*}\right) \leqslant R_{j}\left(r^{*}\right), R_{j+1}\left(r^{*}\right) \leqslant E_{i+1}\left(e^{*}\right)\right)$.

- Region II: $E_{i}\left(e^{*}\right) \geqslant R_{j}\left(r^{*}\right)$ and $E_{i}\left(e^{*}\right) \leqslant R_{j+1}\left(r^{*}\right)\left(\right.$ or $\left.R_{j}\left(r^{*}\right) \leqslant E_{i}\left(e^{*}\right), E_{i+1}\left(e^{*}\right) \leqslant R_{j+1}\left(r^{*}\right)\right)$.

- Region III: $E_{i}\left(e^{*}\right) \geqslant R_{j}\left(r^{*}\right)$ and $E_{i}\left(e^{*}\right) \geqslant R_{j+1}\left(r^{*}\right)\left(\right.$ or $\left.E_{i}\left(e^{*}\right) \geqslant R_{j}\left(r^{*}\right), R_{j+1}\left(r^{*}\right) \geqslant E_{i+1}\left(e^{*}\right)\right)$.

- Region IV: $E_{i}\left(e^{*}\right) \leqslant R_{j}\left(r^{*}\right)$ and $E_{i}\left(e^{*}\right) \geqslant R_{j+1}\left(r^{*}\right)\left(\right.$ or $\left.R_{j}\left(r^{*}\right) \geqslant E_{i}\left(e^{*}\right), E_{i+1}\left(e^{*}\right) \geqslant R_{j+1}\left(r^{*}\right)\right)$.

The relative magnitude of concerned membership values are used in calculating the firing levels of the control rules. For crisp input value $\left(e^{*}, r^{*}\right)$ locating within the $i j$ th-block, the firing levels of the four rules listed in Property 4 in the four regions are shown in Table 1 (see [15]). In obtaining the firing levels, the Zadeh's standard fuzzy intersection ( $\mathrm{min})$ is applied.

Applying the results of Table 1 and Property 3 to Eq. (3), the final crisp output GU $\times u^{*}$ of the USMFs-FLC could be expressed as the sum of a global part, $\mathrm{GU} \times u_{G}^{*}$, and a local part, $\mathrm{GU} \times u_{L}^{*}$. The value of the global part of the crisp output is decided by specific $i j$ th-block in which $\left(e^{*}, r^{*}\right)$ locates and is a constant across the four regions within the $i j$ th-block. The value of local part, on the other hand, varies with the values $e^{*}$ and $r^{*}$ as stated in the following property. 
Table 1

Firing levels of the four rules fired for the four regions in the $i j$ th-block

\begin{tabular}{lllll}
\hline Region & $\phi_{i+1, j+1}(\mathrm{r} 1)$ & $\phi_{i+1, j}(\mathrm{r} 2)$ & $\phi_{i, j+1}(\mathrm{r} 3)$ & $\phi_{i, j}(\mathrm{r} 4)$ \\
\hline I & $R_{j+1}\left(r^{*}\right)$ & $R_{j}\left(r^{*}\right)$ & $E_{i}\left(e^{*}\right)$ & $E_{i}\left(e^{*}\right)$ \\
II & $E_{i+1}\left(e^{*}\right)$ & $R_{j}\left(r^{*}\right)$ & $E_{i}\left(e^{*}\right)$ & $R_{j}\left(r^{*}\right)$ \\
III & $E_{i+1}\left(e^{*}\right)$ & $E_{i+1}\left(e^{*}\right)$ & $R_{j+1}\left(r^{*}\right)$ & $R_{j}\left(r^{*}\right)$ \\
IV & $R_{j+1}\left(r^{*}\right)$ & $E_{i+1}\left(e^{*}\right)$ & $R_{j+1}\left(r^{*}\right)$ & $E_{i}\left(e^{*}\right)$ \\
\hline
\end{tabular}

Property 6. The crisp output of the USMFs-FLC on the ijth-block, $u_{m}=\mathrm{GU} \times u^{*}$, is the sum of a global two-dimensional multilevel relay, $\mathrm{GU} \times u_{G}^{*}$, and a local nonlinear compensator, $\mathrm{GU} \times u_{L}^{*}$ :

$$
\begin{aligned}
& u_{m}=\mathrm{GU} \times u^{*}=\mathrm{GU} \times\left(u_{G}^{*}+u_{L}^{*}\right)=u_{G}+u_{L}, \\
& u_{G}^{*}=\frac{U_{k}^{*}+U_{k+1}^{*}+U_{k+2}^{*}}{3} \quad(k=i+j), \\
& u_{L}^{*}= \begin{cases}\frac{N_{\mathrm{I}, \mathrm{IV}}}{\frac{N_{\mathrm{II}, \mathrm{III}}}{1+2\left(E_{i+1}^{*}-e^{*}\right) /\left(E_{i+1}^{*}-E_{i}^{*}\right)}} & \text { for region } \mathrm{I}, \\
\frac{N_{\mathrm{II}, \mathrm{III}}}{1+2\left(R_{j+1}^{*}-r^{*}\right) /\left(R_{j+1}^{*}-R_{j}^{*}\right)} & \text { for region } \mathrm{II}, \\
\frac{N_{\mathrm{I}, \mathrm{IV}}}{1+2\left(r^{*}-R_{j}^{*}\right) /\left(R_{j+1}^{*}-E_{i}^{*}\right)} & \text { for region } \mathrm{III},\end{cases}
\end{aligned}
$$

where

$$
\begin{aligned}
& N_{\mathrm{I}, \mathrm{IV}}=\left(\frac{r^{*}-R_{j}^{*}}{R_{j+1}^{*}-R_{j}^{*}}\right)\left(\frac{U_{k+3}^{*}-U_{k}^{*}}{3}\right)-\left(\frac{E_{i+1}^{*}-e^{*}}{E_{i+1}^{*}-E_{i}^{*}}\right)\left(\frac{U_{k+2}^{*}-U_{k-1}^{*}}{3}\right), \\
& N_{\mathrm{II}, \mathrm{III}}=\left(\frac{e^{*}-E_{i}^{*}}{E_{i+1}^{*}-E_{i}^{*}}\right)\left(\frac{U_{k+3}^{*}-U_{k}^{*}}{3}\right)-\left(\frac{R_{j+1}^{*}-r^{*}}{R_{j+1}^{*}-R_{j}^{*}}\right)\left(\frac{U_{k+2}^{*}-U_{k-1}^{*}}{3}\right) .
\end{aligned}
$$

Proof. Without loss of generality, suppose the scaled input pattern $\left(e^{*}, r^{*}\right)$ locates in region III of the $i j$ thblock. Then (let $k=i+j$ )

$$
\begin{aligned}
u^{*} & =\frac{\sum_{\phi_{i, j} \neq 0} \phi_{i, j} \bar{u}_{i+j}}{\sum_{\phi_{i, j} \neq 0} \phi_{i, j}}=\frac{\sum_{E_{i}\left(e^{*}\right) \neq 0, R_{j}\left(r^{*}\right) \neq 0} \min \left\{E_{i}\left(e^{*}\right), R_{j}\left(r^{*}\right)\right\} \bar{u}_{i+j}}{\sum_{E_{i}\left(e^{*}\right) \neq 0, R_{j}\left(r^{*}\right) \neq 0} \min \left\{E_{i}\left(e^{*}\right), R_{j}\left(r^{*}\right)\right\}} \\
& =\frac{E_{i+1}\left(e^{*}\right) \bar{u}_{i+j+2}+\left(E_{i+1}\left(e^{*}\right)+R_{j+1}\left(r^{*}\right)\right) \bar{u}_{i+j+1}+R_{j}\left(r^{*}\right) \bar{u}_{i+j}}{E_{i+1}\left(e^{*}\right)+E_{i+1}\left(e^{*}\right)+R_{j+1}\left(r^{*}\right)+R_{j}\left(r^{*}\right)} \\
& =\frac{\left(\frac{e^{*}-E_{i}^{*}}{E_{i+1}^{*}-E_{i}^{*}}\right)\left(\frac{U_{k+1}^{*}+U_{k+2}^{*}+U_{k+3}^{*}}{3}\right)+\left(\frac{e^{*}-E_{i}^{*}}{E_{i+1}^{*}-E_{i}^{*}}+\frac{r^{*}-R_{j}^{*}}{R_{j+1}^{*}-R_{j}^{*}}\right)\left(\frac{U_{k}^{*}+U_{k+1}^{*}+U_{k+2}^{*}}{3}\right)+\left(\frac{R_{j+1}^{*}-r^{*}}{R_{i+1}^{*}-R_{i}^{*}}\right)\left(\frac{U_{k-1}^{*}+U_{k}^{*}+U_{k+1}^{*}}{3}\right)}{2\left(e^{*}-E_{i}^{*}\right) /\left(E_{i+1}^{*}-E_{i}^{*}\right)+1} .
\end{aligned}
$$


Note that

$$
\begin{aligned}
\left(\frac{e^{*}-E_{i}^{*}}{E_{i+1}^{*}-E_{i}^{*}}+\frac{r^{*}-R_{j}^{*}}{R_{j+1}^{*}-R_{j}^{*}}\right) & =\left(2 \frac{e^{*}-E_{i}^{*}}{E_{i+1}^{*}-E_{i}^{*}}+1\right)-\left(\frac{e^{*}-E_{i}^{*}}{E_{i+1}^{*}-E_{i}^{*}}\right)+\left(\frac{r^{*}-R_{j}^{*}}{R_{j+1}^{*}-R_{j}^{*}}-1\right) \\
& =\left(2 \frac{e^{*}-E_{i}^{*}}{E_{i+1}^{*}-E_{i}^{*}}+1\right)-\left(\frac{e^{*}-E_{i}^{*}}{E_{i+1}^{*}-E_{i}^{*}}\right)-\left(\frac{R_{j+1}^{*}-r^{*}}{R_{j+1}^{*}-R_{j}^{*}}\right) .
\end{aligned}
$$

Thus,

$$
\begin{aligned}
u^{*}= & \frac{U_{k}^{*}+U_{k+1}^{*}+U_{k+2}^{*}}{3} \\
& +\frac{\left(\frac{e^{*}-E_{i}^{*}}{E_{i+1}^{*}-E_{i}^{*}}\right)\left(\frac{U_{k+1}^{*}+U_{k+2}^{*}+U_{k+3}^{*}}{3}-\frac{U_{k}^{*}+U_{k+1}^{*}+U_{k+2}^{*}}{3}\right)+\left(\frac{R_{j+1}^{*}-r^{*}}{R_{j+1}^{*}-R_{i}^{*}}\right)\left(\frac{U_{k-1}^{*}+U_{k}^{*}+U_{k+1}^{*}}{3}-\frac{\left.U_{k}^{*}+U_{k+1}^{*}+U_{k+2}^{*}\right)}{3}\right.}{2\left(e^{*}-E_{i}^{*}\right) /\left(E_{i+1}^{*}-E_{i}^{*}\right)+1} \\
= & \frac{U_{k}^{*}+U_{k+1}^{*}+U_{k+2}^{*}}{3}+\frac{\left(\frac{e^{*}-E_{i}^{*}}{E_{i+1}^{*}-E_{i}^{*}}\right)\left(\frac{U_{k+3}^{*}-U_{k}^{*}}{3}\right)-\left(\frac{R_{j+1}^{*}-r^{*}}{R_{i+1}^{*}-R_{i}^{*}}\right)\left(\frac{U_{k+2}^{*}-U_{k-1}^{*}}{3}\right)}{2\left(e^{*}-E_{i}^{*}\right) /\left(E_{i+1}^{*}-E_{i}^{*}\right)+1} \\
& \equiv u_{G}^{*}+u_{L}^{*} .
\end{aligned}
$$

From Property 6, one can find that within a specific $i j$ th-block the output of the USMFs-FLC consists of two parts [15]: the global term is a constant which is determined by location of the $i j$ th-block and is independent of the values of $e^{*}$ and $r^{*}$ within the block; the local part is a nonlinear relation of values of $e^{*}$ and $r^{*}$. The USMFs-FLC discussed above thus can be viewed as the combination of a two-dimensional multilevel relay (the global term) and a nonlinear compensator (the local term), and the latter will be further discussed in the subsequent subsection.

The effects of distributions of principal values for $e, r$, and $u$, on final control response, including global and local parts, respectively, can be investigated furthermore. Without loss of generality, such discussions would be illustrated for SSMFs-FLC and ESMFs-FLC only. According to the definition of the principal values $E_{i}^{*}$ 's, $R_{j}^{*}$, s, and $U_{k}^{*}$ 's in the SSMFs, the effects of the shrinking factors can be addressed in two respects.

For input variable of the SSMFs-FLC, the values of $s_{e}$ and $s_{r}$ influence both the location and shape of the $i j$ th-block in the domain $U_{e} \times U_{r}$. Owing to this, a specific set of $\left(e^{*}, r^{*}\right)$ may locate in different $i j$ th-block for different $s_{e}$ and $s_{r}$ values and thus may fire distinct control rules in obtaining the final output. As for the output variable $u$, different effects are induced for $u_{G}^{*}$ and $u_{L}^{*}$ by changing the shrinking factor $s_{u}$. For $u_{G}^{*}$, the modification of $s_{u}$ changes its magnitude only; but for $u_{L}^{*}$, both magnitude and nonlinearity are altered by varying the value of $s_{u}$. Figs. 3-5 elucidate the effects of shrinking factors on final, global, and local control outputs, respectively. The effects of the shrinking factors on control performance for a wide variety of linear/nonlinear processes can be found elsewhere [2].

The following property states the upper and lower bounds of the local output and the final control actions, which are relevant to further discussion about ultimate control behavior as the number of linguistic terms becomes infinity. 

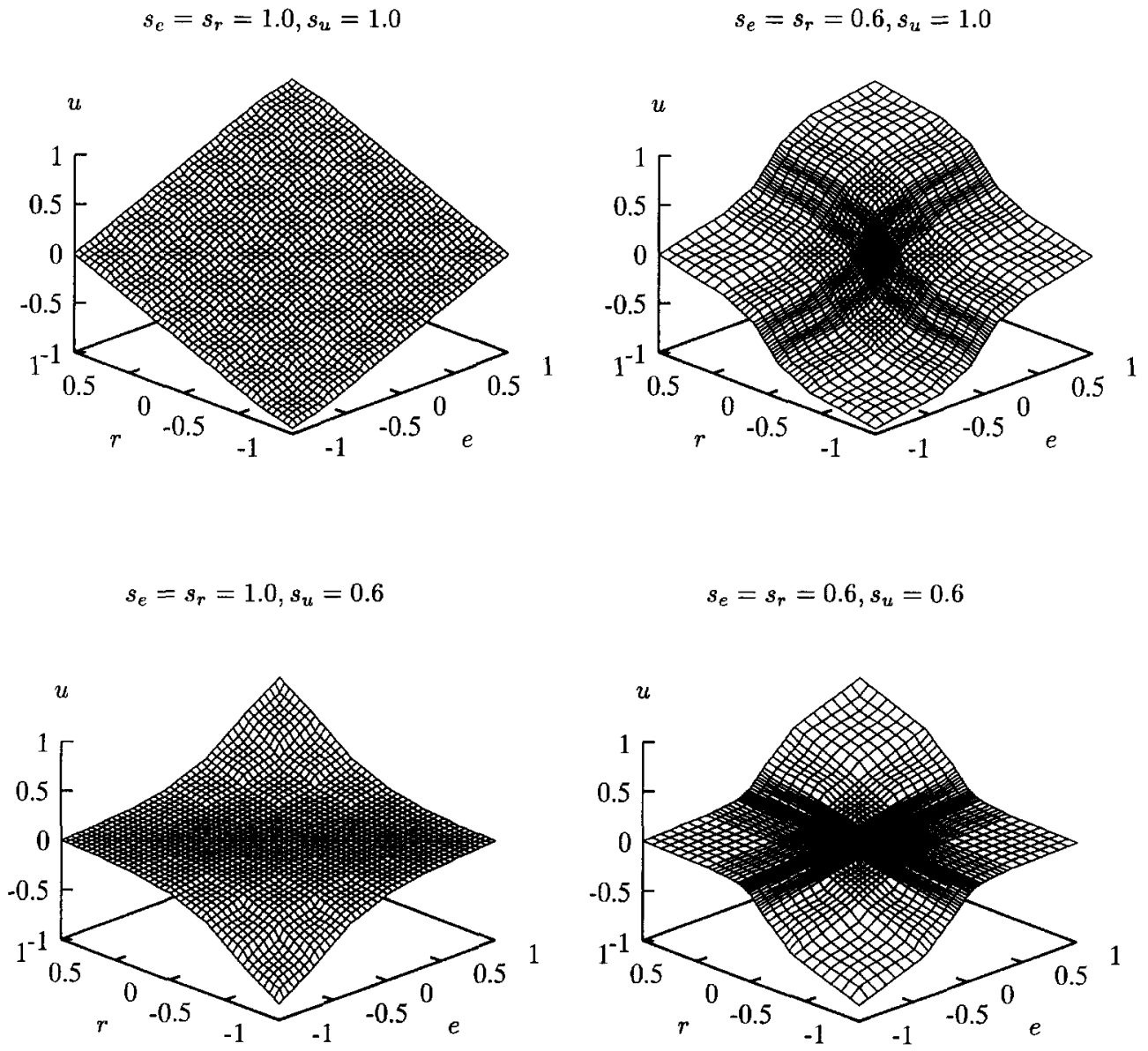

Fig. 3. Effects of shrinking factors on final control outputs of the SSMFs-FLC $\left(m_{e}=m_{r}=3\right)$.

Property 7. The local output term and the final crisp output of the USMFS-FLC on the ijth-block is, respectively, bounded by $(k=i+j)$

$$
-\mathrm{GU} \times \frac{U_{k+2}^{*}-U_{k-1}^{*}}{3} \leqslant \mathrm{GU} \times u_{L}^{*}=u_{L} \leqslant \mathrm{GU} \times \frac{U_{k+3}^{*}-U_{k}^{*}}{3} .
$$

and

$$
\mathrm{GU} \times \frac{U_{k-1}^{*}+U_{k}^{*}+U_{k+1}^{*}}{3} \leqslant \mathrm{GU} \times u^{*}=u_{m} \leqslant \mathrm{GU} \times \frac{U_{k+1}^{*}+U_{k+2}^{*}+U_{k+3}^{*}}{3},
$$

where the upper (lower) bound for final output on the ijth-block is equivalent to the global output on the $(i+1, j)$ th-block and $(i, j+1)$ th-block $((i-1, j)$ th-block and $(i, j-1)$ th-block $)$.

Proof. Substituting lower and upper bounds for $e^{*}$ and $r^{*}$ for each region on the $i j$ th-block into local output equations in Property 6 (on region I, for example, it is $e^{*} \in \frac{1}{2}\left[\left(E_{i}^{*}+E_{i+1}^{*}\right), E_{i+1}^{*}\right]$ and $r^{*} \in\left[R_{j}^{*}, R_{j+1}^{*}\right]$ ) leads to $-\frac{1}{3}\left(U_{k+2}^{*}-U_{k-1}^{*}\right) \leqslant u_{L}^{*} \leqslant \frac{1}{3}\left(U_{k+3}^{*}-U_{k}^{*}\right)$. The lower/upper bounds for final output are obtained by adding up the scope of local output with global output. 

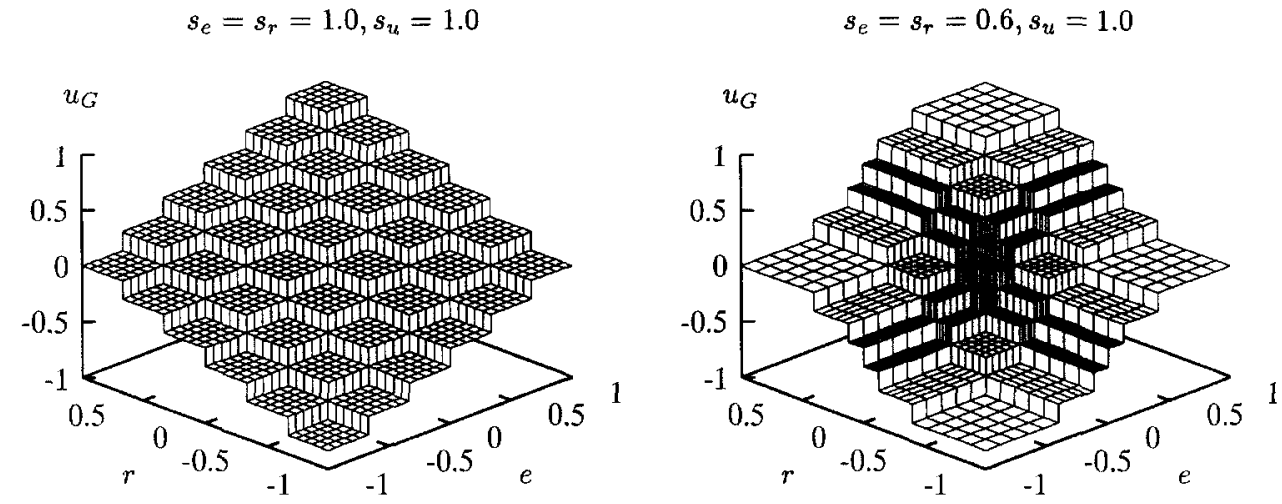

$$
s_{e}=s_{r}=1.0, s_{u}=0.6
$$

$$
s_{e}=s_{r}=0.6, s_{u}=0.6
$$
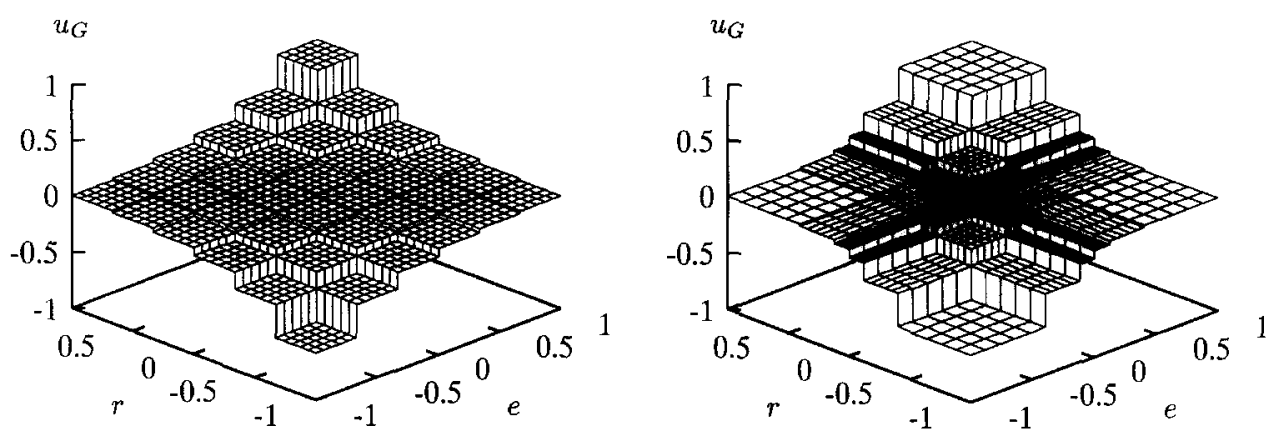

Fig. 4. Effects of shrinking factors on global outputs of the SSMFs-FLC $\left(m_{e}=m_{r}=3\right.$ ).

Property 6 implies that the USMFs-FLC is nonlinear in nature. The degree of nonlinearity of the USMFsFLC can be measured from two aspects: the limited resolution of the global multilevel relay and the positiondependent nonlinear local output. Obviously, increasing the number of linguistic terms would increase the degree of resolution of the multilevel relay and, thus, would decrease its contribution to nonlinearity due to the assumption of simple control rules that we have made to simplify the analysis. The output shrinking factor is another element affecting the resolution and the nonlinearity. A natural measure of nonlinear degree can thus be defined as the maximum difference of the multilevel relay between adjacent blocks. Obviously, the average value of differences between adjacent multilevel relays can also be used as the nonlinearity measure. We adopt the former definition for simplicity. Notably, the maximum magnitude of local output can also be interpreted as the degree of nonlinearity of the controller, since the role of local output is to compensate the global one so as to lead to a continuous final output. This fact leads to the following property concerning the measure of nonlinearity.

Property 8. The degree of nonlinearity of the USMFS-FLC, SSMFS-FLC, and ESMFS-FLC, defined as the maximum difference of adjacent global outputs or the maximum value of local output on $U_{e} \times U_{r}$, are

$$
\varrho \equiv \max _{\substack{\forall i, j \in I_{m}-\{m\} \\ i+j \geqslant-2 m+1}}\left|u_{G}^{*}(i+j)-u_{G}^{*}(i+j-1)\right|
$$




$$
s_{e}=s_{r}=1.0, s_{u}=1.0
$$

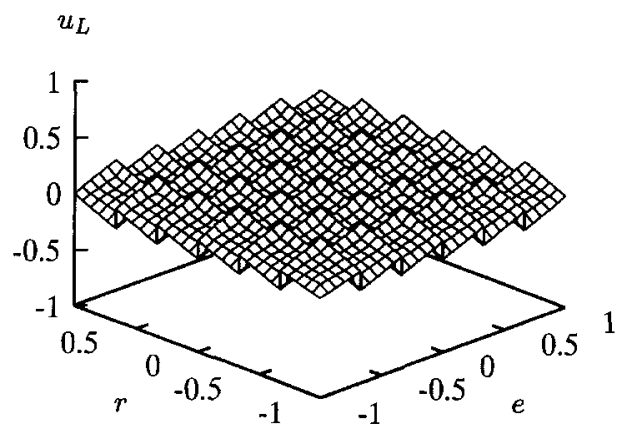

$$
s_{e}=s_{r}=1.0, s_{u}=0.6
$$

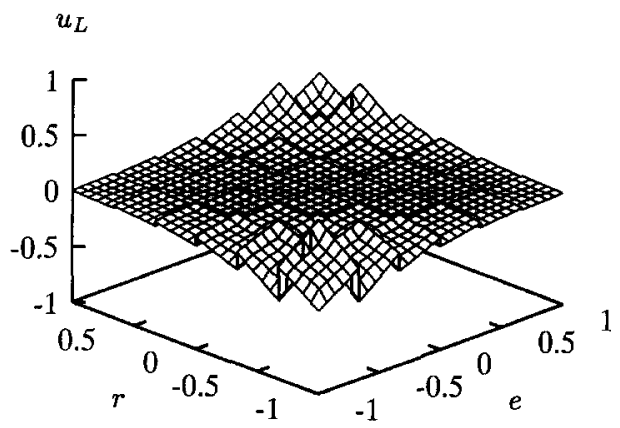

$s_{e}=s_{r}=0.6, s_{u}=1.0$

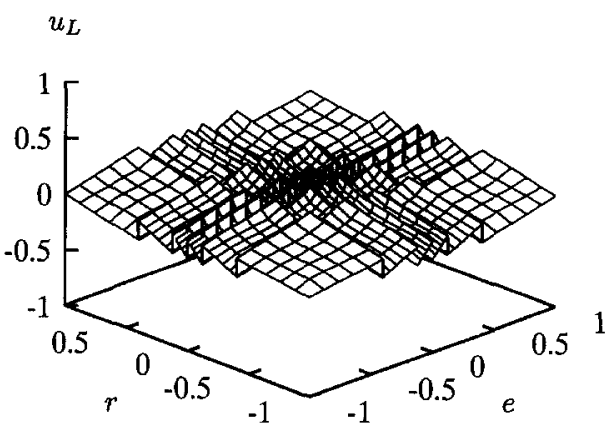

$s_{e}=s_{r}=0.6, s_{u}=0.6$

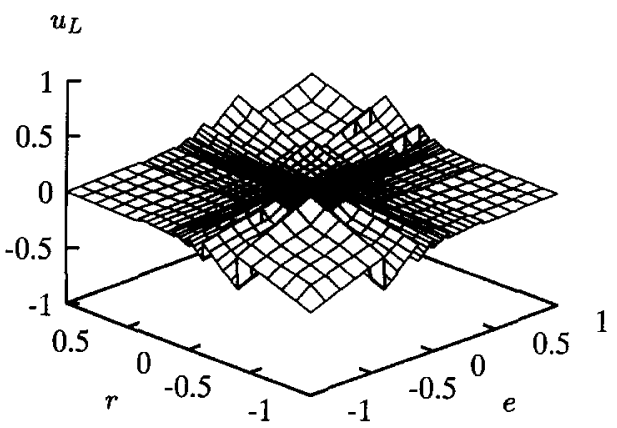

Fig. 5. Effects of shrinking factors on local outputs of the SSMFs-FLC $\left(m_{e}=m_{r}=3\right.$ ).

$$
=\max _{\substack{\forall i, j \in I_{m}-\{m\} \\ i+j \geqslant-2 m+1}}\left|u_{L}^{*}\right|= \begin{cases}\max _{\forall i, j \in I_{m}-\{m\}} \frac{U_{i+j+2}^{*}-U_{i+j-1}^{*}}{3} & \text { for USMFs-FLC, } \\ \frac{1-(1-3 / 2 m)}{3} \times \begin{cases}s_{u}(m=1) \\ s_{u}^{3}(m \geqslant 2)\end{cases} & \text { for SSMFs-FLC, } \\ \frac{1}{2 m} & \text { for ESMFs-FLC. }\end{cases}
$$

Proof. The definition of degree of nonlinearity leads straightforwardly to this property.

From the definition of $\varrho$, the higher the value of $\varrho$ the higher the degree of nonlinearity, and $\varrho=0$ indicates the FLC a linear controller. Figs. 6 and 7 illustrate the effect of the number of linguistic terms on resolution of $u_{G}^{*}$ and on the effective ranges of $u_{L}^{*}$ for SSMFs-FLC and ESMFs-FLC. The degree of nonlinearities are also shown in these figures. Fig. 8 shows the effects of linguistic numbers and output shrinking factor on degree-of-nonlinearity for the SSMFs-FLC. These illustrations and Property 8 implies that SSMFs-FLC would behave with limited nonlinearity depending on the magnitude of output shrinking factor, and ESMFs-FLC 
$m=1, \varrho=0.433$
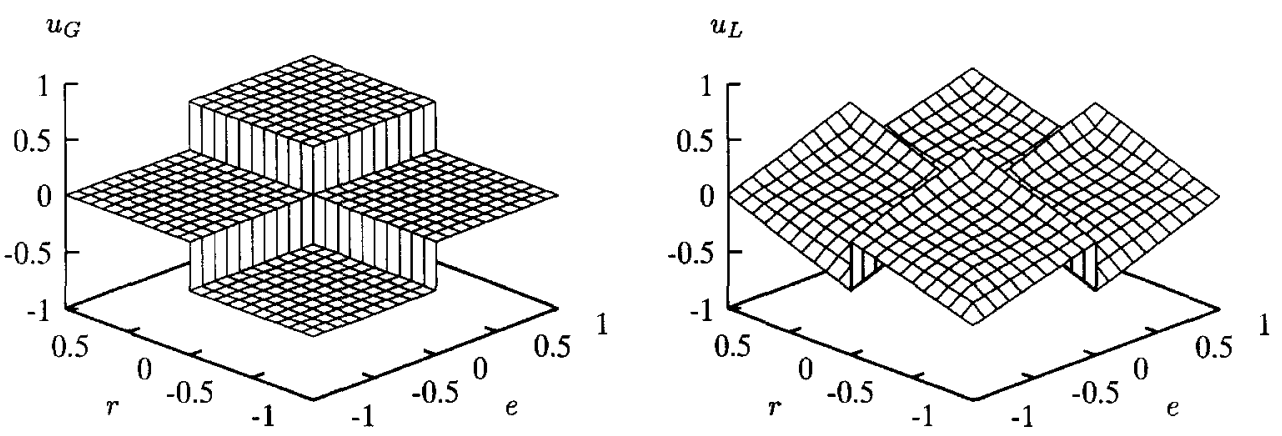

$m=2, \varrho=0.315$
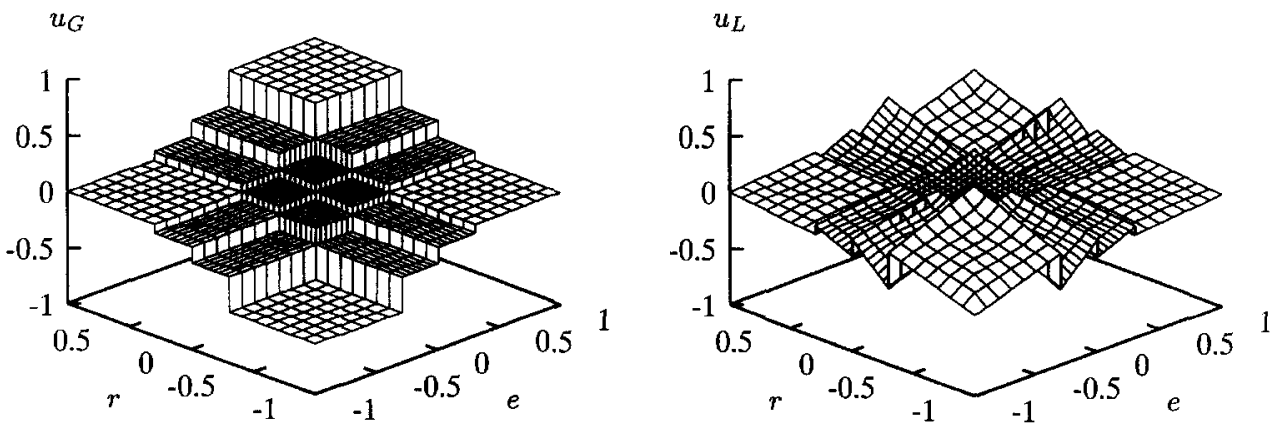

$m=4, \varrho=0.288$
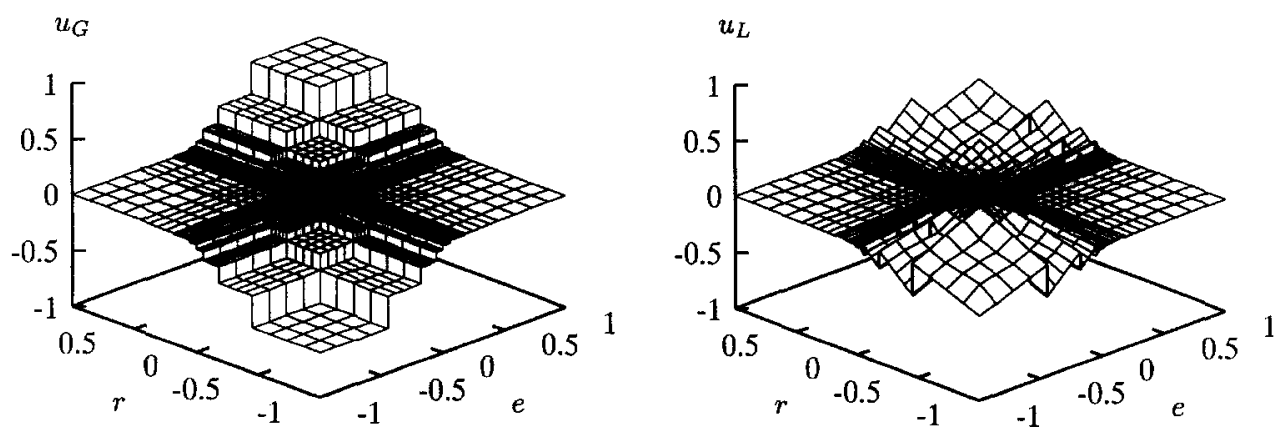

Fig. 6. Effects of linguistic terms on global (left) and local (right) outputs and degree-of-nonlinearity of the SSMFs-FLC $\left(s_{e}=s_{r}=s_{u}=0.6 ; \varrho\left(m \rightarrow \infty, s_{u}=0.6\right)=0.216\right)$.

would become a linear controller as $m \rightarrow \infty$. That is,

$$
\varrho \stackrel{m \rightarrow \infty}{\longrightarrow} \begin{cases}1-s_{u}^{3} / 3 & \text { for SSMFs-FLC } \\ 0 & \text { for ESMFs-FLC }\end{cases}
$$




$$
m=1, \varrho=0.500
$$
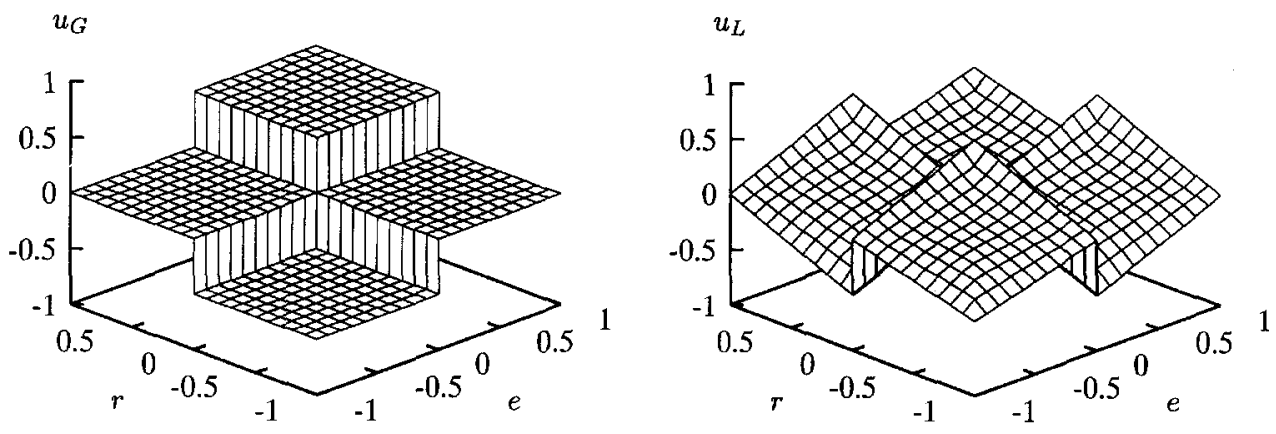

$$
m=2, \varrho=0.250
$$
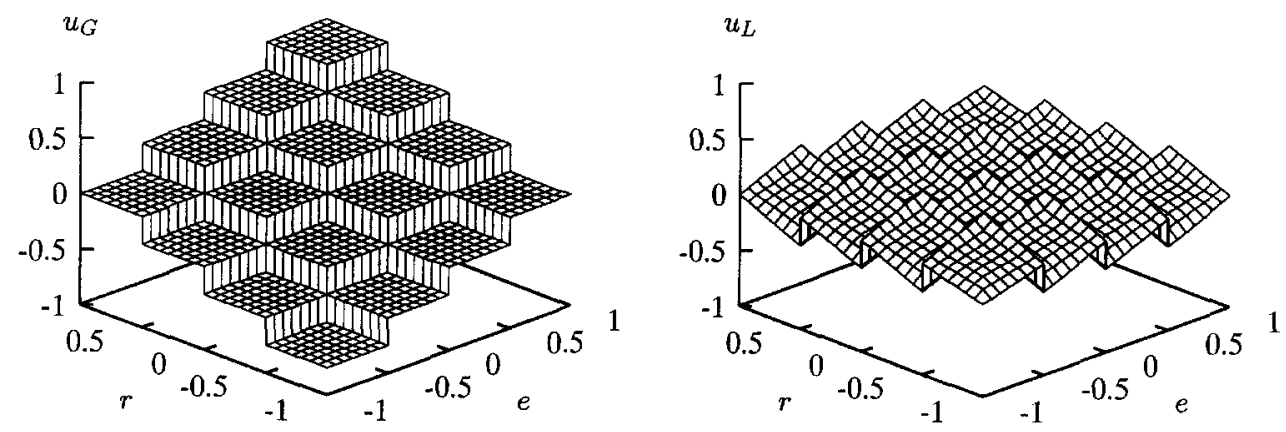

$$
m=4, \varrho=0.125
$$
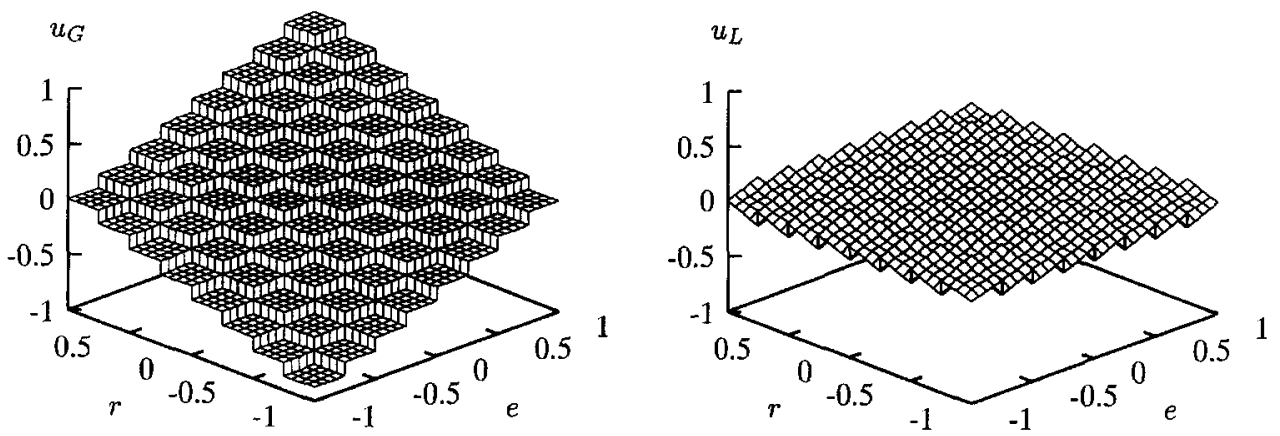

Fig. 7. Effects of linguistic terms on global (left) and local (right) outputs and degree-of-nonlinearity of the ESMFs-FLC $(\varrho(m \rightarrow \infty)=0)$.

The following two properties further point out the ultimate behavior of SSMFs-FLC and ESMFs-FLC, respectively, as $m \rightarrow \infty$. Note that the linear nature of these FLCs as $m \rightarrow \infty$ comes from the assumption of simple control rules. 


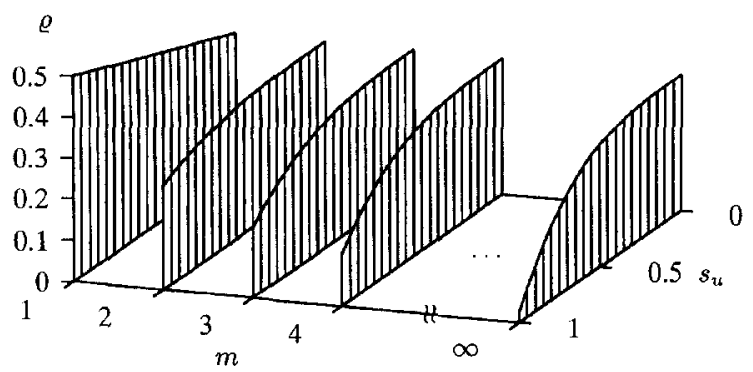

Fig. 8. Effects of linguistic numbers $(m)$ and output shrinking factor $\left(s_{u}\right)$ on degree-of-nonlinearity $(\varrho)$ for SSMFs-FLC where $\rho\left(m, s_{u}=0\right)=\frac{1}{3}$.

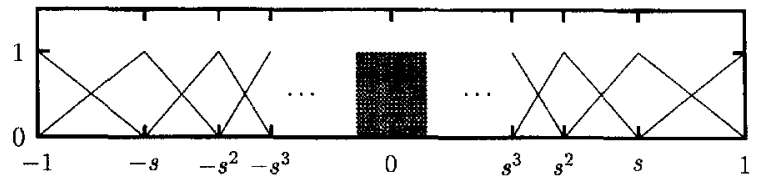

(a)

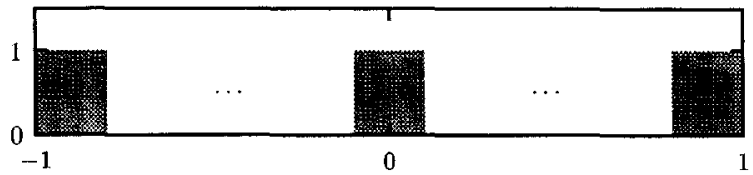

(b)

Fig. 9. The ultimate distribution of membership functions as $m \rightarrow \infty$ for (a) SSMFs-FLC and (b) ESMFs-FLC. The shaded area is composed of infinite number of closely-spaced triangular MFs.

Property 9. The ultimate distribution of membership functions of an SSMFS-FLC with infinite number of linguistic terms is shown in Fig. 9(a). And the crisp output of such an SSMFs-FLC on the ijth-block are bounded by two linear PI controllers,

$$
K_{p}^{\prime}\left(r_{m}+\frac{T}{\tau_{l}^{\prime}} e_{m}\right) \leqslant u_{m} \leqslant K_{p}^{u}\left(r_{m}+\frac{T}{\tau_{I}^{u}} e_{m}\right)
$$

where

$$
\begin{aligned}
& K_{p}^{f}=\mathrm{GU} \times \mathrm{GR} \times \frac{s_{r}^{-m+|j+1|}}{6}\left(s_{u}^{2 m-|i+j-1|}+s_{u}^{2 m-|i+j|}+s_{u}^{2 m-|i+j+1|}\right), \\
& K_{p}^{u}=\mathrm{GU} \times \mathrm{GR} \times \frac{s_{r}^{-m+|j|}}{6}\left(s_{u}^{2 m-|i+j+1|}+s_{u}^{2 m-|i+j+2|}+s_{u}^{2 m-|i+j+3|}\right), \\
& \tau_{I}^{f}=\frac{\mathrm{GR}}{\mathrm{GE}} \frac{s_{r}^{-m+|j+1|}}{s_{e}^{-m+|i+1|}} T, \quad \tau_{I}^{u}=\frac{\mathrm{GR}}{\mathrm{GE}} \frac{s_{r}^{-m+|j|}}{s_{e}^{-m+|i|}} T .
\end{aligned}
$$

Proof. From the definition of SSMFs, $E_{i}^{*}=(i / m) s_{e}^{m-|i|}$, one has $E_{m}^{*}=1$, and the second principal value from the right limit of $U_{e}$ is $E_{m-1}^{*}=((m-1) / m) s_{e} \approx s_{e}$ as $m \rightarrow \infty$. Similarly, $E_{m-2}^{*}=((m-2) / m) s_{e}^{2} \approx s_{e}^{2}$, and 
$E_{m-3}^{*}=((m-3) / m) s_{e}^{3} \approx s_{e}^{3}$, etc. Similar derivations leads to Fig. 9(a) where the shaded area is composed of infinite number of closely spaced triangles.

Suppose $e^{*} \in\left[E_{i}^{*}, E_{i+1}^{*}\right]$. Then, $(i / m) s_{e}^{m-|i|}=E_{i}^{*} \leqslant e^{*} \leqslant E_{i+1}^{*}=((i+1) / m) s_{e}^{m-|i+1|} \approx(i / m) s_{e}^{m-|i+1|}$ as $m \rightarrow \infty$. Thus, $e^{*} s_{e}^{-m+|i+1|} \leqslant i / m \leqslant e^{*} s_{e}^{-m+|i|}$, and similarly $r^{*} s_{r}^{-m+|j+1|} \leqslant j / m \leqslant r^{*} s_{r}^{-m+|j|}$. Taking definition for $U_{k}^{*}$ 's and the upper bound for crisp output into equation in Property 7 ,

$$
\begin{aligned}
& u_{m} \leqslant \mathrm{GU} \times \frac{U_{i+j+1}^{*}+U_{i+j+2}^{*}+U_{i+j+3}^{*}}{3} \\
& =\frac{\mathrm{GU}}{3} \times\left(\frac{i+j+1}{2 m} s_{u}^{2 m-|i+j+1|}+\frac{i+j+2}{2 m} s_{u}^{2 m-|i+j+2|}+\frac{i+j+3}{2 m} s_{u}^{2 m-|i+j+3|}\right) \\
& =\frac{\mathrm{GU}}{6} \times\left(s_{u}^{2 m-|i+j+1|}\left(e^{*} s_{e}^{-m+|i|}+r^{*} s_{r}^{-m+|j|}+\frac{1}{m}\right)\right. \\
& \left.+s_{u}^{2 m-|i+j+2|}\left(e^{*} s_{e}^{-m+|i|}+r^{*} s_{r}^{-m+|j|}+\frac{2}{m}\right)+s_{u}^{2 m-|i+j+3|}\left(e^{*} s_{e}^{-m+|i|}+r^{*} s_{r}^{-m+|j|}+\frac{3}{m}\right)\right) \\
& \approx \frac{\mathrm{GU}}{6} \times\left(s_{u}^{2 m-|i+j+1|}+s_{u}^{2 m-|i+j+2|}+s_{u}^{2 m-|i+j+3|}\right)\left(e^{*} s_{e}^{-m+|i|}+r^{*} s_{r}^{-m+|j|}\right) \quad \text { as } m \rightarrow \infty \\
& =\frac{\mathrm{GU} \times \mathrm{GR} \times s_{r}^{-m+|j|}}{6} \times\left(s_{u}^{2 m-|i+j+1|}+s_{u}^{2 m-|i+j+2|}+s_{u}^{2 m-|i+j+3|}\right)\left(r_{m}+\left(T / \frac{\mathrm{GR}}{\mathrm{GE}} \frac{s_{r}^{-m+|j|}}{s_{e}^{-m+|i|}} T\right) e_{m}\right) \\
& \equiv K_{p}^{u}\left(r_{m}+\frac{T}{\tau_{I}^{u}} e_{m}\right)
\end{aligned}
$$

Similar derivation can find $K_{p}^{\ell}$ and $\tau_{I}^{\ell}$.

Property 10 (Ying [15]). The ultimate distribution of membership functions of an ESMFs-FLC with infinite number of linguistic terms is shown in Fig. 9(b), and such an ESMFs-FLC is equivalent to a linear PI controller,

$$
u_{m}=\frac{\mathrm{GU} \times \mathrm{GR}}{2}\left(r_{m}+\frac{T}{(\mathrm{GR} / \mathrm{GE}) T} e_{m}\right) \quad \text { as } m \rightarrow \infty .
$$

Proof. The space between adjacent principal values is $E_{i+1}^{*}-E_{i}^{*}=1 / m \rightarrow 0$ as $m \rightarrow \infty$. This results in an infinite number of closely spaced triangles such as shown in the figure. Furthermore, substituting $s_{e}=s_{r}=s_{u}=1$ into equations in Property 9 leads to the linear controller.

Properties 9 and 10 imply that, under the assumption of simple rule base, increasing the number of linguistic terms in the USMFs-FLC will result in a quasi-linear PI controller. The control output of an USMFs-FLC is kept within two linear PI controllers, and it is in fact equivalent to a linear PI controller if its membership functions are equally spaced triangular fuzzy partitions. Obviously, these properties come from the assumption of simple rule mapping. Thus, one cannot conclude that the linearity of the real-world FLCs is proportional to the number of linguistic terms, since the assumption of simple rules is not natural in most practical applications. We take this assumption just because it will simplify the subsequent mathematical analysis. 
Table 2

\begin{tabular}{lll}
\hline & $\frac{K_{e}}{\mathrm{GU}}$ & $\frac{K_{r}}{\mathrm{GU}}$ \\
\hline Region I & $\frac{\left(U_{k+2}^{*}-U_{k-1}^{*}\right) / 3}{1+2\left(E_{i+1}^{*}-e^{*}\right) /\left(E_{i+1}^{*}-E_{i}^{*}\right)}$ & $\frac{\left(U_{k+3}^{*}-U_{k}^{*}\right) / 3}{1+2\left(E_{i+1}^{*}-e^{*}\right) /\left(E_{i+1}^{*}-E_{i}^{*}\right)}$ \\
Region II & $\frac{\left(U_{k+3}^{*}-U_{k}^{*}\right) / 3}{1+2\left(R_{j+1}^{*}-r^{*}\right) /\left(R_{j+1}^{*}-R_{i}^{*}\right)}$ & $\frac{\left(U_{k+2}^{*}-U_{k-1}^{*}\right) / 3}{1+2\left(R_{j+1}^{*}-r^{*}\right) /\left(R_{j+1}^{*}-R_{i}^{*}\right)}$ \\
Region III & $\frac{\left(U_{k+3}^{*}-U_{k}^{*}\right) / 3}{1+2\left(e^{*}-E_{i}^{*}\right) /\left(E_{i+1}^{*}-E_{i}^{*}\right)}$ & $\frac{\left(U_{k+2}^{*}-U_{k-1}^{*}\right) / 3}{1+2\left(e^{*}-E_{i}^{*}\right) /\left(E_{i+1}^{*}-E_{i}^{*}\right)}$ \\
Region IV & $\frac{\left(U_{k+2}^{*}-U_{k-1}^{*}\right) / 3}{1+2\left(r^{*}-R_{j}^{*}\right) /\left(R_{j+1}^{*}-R_{i}^{*}\right)}$ & $\frac{\left(U_{k+3}^{*}-U_{k}^{*}\right) / 3}{1+2\left(r^{*}-R_{j}^{*}\right) /\left(R_{j+1}^{*}-R_{i}^{*}\right)}$ \\
\hline
\end{tabular}

\subsection{Interpreting local output of the USMFS-FLC as a nonlinear compensator}

It has been shown in Property 7 that the local output in the $i j$ th-block is limited within $-\frac{1}{3}\left(U_{k+2}^{*}-U_{k-1}^{*}\right) \leqslant$ $u_{L}^{*} \leqslant \frac{1}{3}\left(U_{k+3}^{*}-U_{k}^{*}\right)$. The role of local part is to complement the action of the global multilevel relay to a nonlinear yet continuous final crisp output. There exists at least one reference point; thus the local compensation becomes zero and the final crisp output equals to the global one. The amount of compensation for $u_{L}^{*}$ would then be proportional to relative position of current input, $\left(e^{*}, r^{*}\right)$, to the reference point. This fact is stated in the following property.

Property 11. The local part of the final crisp output of the USMFS-FLC on the ijth-block can be viewed as a local nonlinear compensator of the form

$$
\mathrm{GU} \times u_{L}=K_{e}\left(\frac{e^{*}-e^{0}}{E_{i+1}^{*}-E_{i}^{*}}\right)+K_{r}\left(\frac{r^{*}-r^{0}}{R_{j+1}^{*}-R_{j}^{*}}\right),
$$

where

$$
e^{0}=\frac{E_{i+1}^{*} \Delta+E_{i}^{*}}{1+\Delta}, \quad r^{0}=\frac{R_{j+1}^{*} \Delta+R_{j}^{*}}{1+\Delta}, \quad \Delta \equiv \frac{U_{k+2}^{*}-U_{k-1}^{*}}{U_{k+3}^{*}-U_{k}^{*}} .
$$

Here, $\left(e^{0}, r^{0}\right)$ is a reference point which is the intersection of two zero local output contours on either regions I and II or regions IV and III of the ijth-block, and thus $u_{L}^{*}\left(e^{*}=e^{0}, r^{*}=r^{0}\right)=0 . K_{e}$ and $K_{r}$ are the local proportional gains of the local nonlinear compensator of the form listed in Table 2.

Proof. From Property 6, there exist two zero contours for local output,

$$
\begin{aligned}
& N_{\mathrm{I}, \mathrm{IV}}=\left(\frac{r^{*}-R_{j}^{*}}{R_{j+1}^{*}-R_{j}^{*}}\right)\left(\frac{U_{k+3}^{*}-U_{k}^{*}}{3}\right)-\left(\frac{E_{i+1}^{*}-e^{*}}{E_{i+1}^{*}-E_{i}^{*}}\right)\left(\frac{U_{k+2}^{*}-U_{k-1}^{*}}{3}\right)=0 \text { on region I or IV, } \\
& N_{\mathrm{II}, \mathrm{III}}=\left(\frac{e^{*}-E_{i}^{*}}{E_{i+1}^{*}-E_{i}^{*}}\right)\left(\frac{U_{k+3}^{*}-U_{k}^{*}}{3}\right)-\left(\frac{R_{j+1}^{*}-r^{*}}{R_{j+1}^{*}-R_{j}^{*}}\right)\left(\frac{U_{k+2}^{*}-U_{k-1}^{*}}{3}\right)=0 \text { on region II or III. }
\end{aligned}
$$


Solving these two zero contour lines results in $e^{0}$ and $r^{0}$. The local output then becomes (for region I as an illustration):

$$
\begin{aligned}
& u_{L}^{*}=\frac{N_{\mathrm{I}, \mathrm{IV}}}{1+2\left(E_{i+1}^{*}-e^{*}\right) /\left(E_{i+1}^{*}-E_{i}^{*}\right)} \\
& =\frac{\left(\frac{\left(r^{*}-r^{0}\right)+\left(r^{0}-R_{j}^{*}\right)}{R_{i+1}^{*}-R_{j}^{*}}\right)\left(\frac{U_{k+3}^{*}-U_{k}^{*}}{3}\right)-\left(\frac{\left(E_{i+1}^{*}-e^{0}\right)+\left(e^{0}-e^{*}\right)}{E_{i+1}^{*}-E_{i}^{*}}\right)\left(\frac{U_{k+2}^{*}-U_{k-1}^{*}}{3}\right)}{1+2\left(E_{i+1}^{*}-e^{*}\right) /\left(E_{i+1}^{*}-E_{i}^{*}\right)} \\
& =\frac{\left(\frac{r^{*}-r^{0}}{R_{j+1}^{*}-R_{i}^{*}}\right)\left(\frac{U_{k+3}^{*}-U_{k}^{*}}{3}\right)-\left(\frac{e^{0}-e^{*}}{E_{i+1}^{*}-E_{i}^{*}}\right)\left(\frac{U_{k+2}^{*}-U_{k-1}^{*}}{3}\right)}{1+2\left(E_{i+1}^{*}-e^{*}\right) /\left(E_{i+1}^{*}-E_{i}^{*}\right)} \\
& +\underbrace{\frac{\left(\frac{r^{0}-R_{j}^{*}}{R_{j+1}^{*}-R_{j}^{*}}\right)\left(\frac{U_{k+3}^{*}-U_{k}^{*}}{3}\right)-\left(\frac{E_{i+1}^{*}-e^{0}}{E_{i+1}^{*}-E_{i}^{*}}\right)\left(\frac{U_{k+2}^{*}-U_{k-1}^{*}}{3}\right)}{1+2\left(E_{i+1}^{*}-e^{*}\right) /\left(E_{i+1}^{*}-E_{i}^{*}\right)}}_{=0} \\
& =\frac{\left(U_{k+2}^{*}-U_{k-1}^{*}\right) / 3}{1+2\left(E_{i+1}^{*}-e^{*}\right) /\left(E_{i+1}^{*}-E_{i}^{*}\right)}\left(\frac{e^{*}-e^{0}}{E_{i+1}^{*}-E_{i}^{*}}\right)+\frac{\left(U_{k+3}^{*}-U_{k}^{*}\right) / 3}{1+2\left(E_{i+1}^{*}-e^{*}\right) /\left(E_{i+1}^{*}-E_{i}^{*}\right)}\left(\frac{r^{*}-r^{0}}{R_{j+1}^{*}-R_{j}^{*}}\right) .
\end{aligned}
$$

It is noted that the farther the input pattern is from the middle point of the $i j$ th-block the larger the two proportional gains. Furthermore, the ranges of these two local proportional gains can be derived by substituting proper $e^{*}$ and $r^{*}$ into the equations listed in Property 11 such as stated in what follows.

Property 12. The ranges of local proportional gains of the USMFS-FLC on the ijth-block are $(k=i+j)$

for regions I, IV: $\frac{1}{2} \leqslant\left(\frac{K_{e} / \mathrm{GU}}{\left(U_{k+2}^{*}-U_{k-1}^{*}\right) / 3}\right) \leqslant 1 ; \quad \frac{1}{2} \leqslant\left(\frac{K_{r} / \mathrm{GU}}{\left(U_{k+3}^{*}-U_{k}^{*}\right) / 3}\right) \leqslant 1$,

for regions II, III: $\frac{1}{2} \leqslant\left(\frac{K_{e} / \mathrm{GU}}{\left(U_{k+3}^{*}-U_{k}^{*}\right) / 3}\right) \leqslant 1 ; \quad \frac{1}{2} \leqslant\left(\frac{K_{r} / \mathrm{GU}}{\left(U_{k+2}^{*}-U_{k-1}^{*}\right) / 3}\right) \leqslant 1$.

Proof. One can find the results straightforwardly by substituting the ranges of $e^{*}$ and $r^{*}$ for each region into equations in Property 11.

\subsection{Local stability analysis for PI-type FLCs}

The feedback control system with USMFs-FLC (and SSMFs-FLC, ESMFs-FLC) as the controller is nonlinear for either linear or nonlinear processes. Assume fuzzy feedback control system is autonomous (time-invariant), and let $\lambda_{i}$ 's denote eigenvalues of the Jacobian matrix of the nonlinear fuzzy feedback system evaluated at equilibrium point (set point). By using the Lyapunov's linearization method on stability [5,11], the fuzzy-logic-based control system is asymptotically stable at the equilibrium point if all eigenvalues are negative in real part. Since the linearized fuzzy controller would be corresponding to a linear $\mathrm{PI}$ one, the inclined degrees of the tangent plane defined in both $e$ and $r$, the inputs of the FLCs, directions around the desired equilibrium point is investigated. The following properties state the local stability 
criteria for a feedback control system with USMFs-FLC, SSMFs-FLC, and ESMFs-FLC, respectively, as the controller.

Property 13. For a given process, linear or nonlinear, and an USMFs-FLC with $U_{0}^{*}=0$ and $U_{-1}^{*}=-U_{1}^{*}$, the fuzzy feedback control system is asymptotically stable at the equilibrium point (set point) if and only if the linear PI control system either with $K_{p}=\mathrm{GU} \times \mathrm{GR} \times \frac{1}{3}\left(U_{2}^{*}-U_{-1}^{*}\right) / R_{1}^{*}$ and $\tau_{I}=\mathrm{GR} / \mathrm{GE} \times-E_{-1}^{*} / R_{1}^{*} \times$ $\left(\left(U_{2}^{*}-U_{-1}^{*}\right) /\left(U_{1}^{*}-U_{-2}^{*}\right)\right) T$ or with $K_{p}=\mathrm{GU} \times \mathrm{GR} \times \frac{1}{3}\left(U_{1}^{*}-U_{-2}^{*}\right) /-R_{-1}^{*}$ and $\tau_{I}=\mathrm{GR} / \mathrm{GE} \times E_{1}^{*} /-R_{-1}^{*} \times$ $\left(\left(U_{1}^{*}-U_{-2}^{*}\right) /\left(U_{2}^{*}-U_{-1}^{*}\right)\right) T$ is asymptotically stable.

Proof. The process variable would reach the equilibrium point $(e=r=0)$ according to locus where $e$ and $r$ have opposite signs. This means the input pattern would be on region I or IV of the $(-1,0)$ th-block or on region II or III of the $(0,-1)$ th-block. The global output for both cases is zero,

$$
\mathrm{GU} \times u_{G}^{*}=\mathrm{GU} \times \frac{U_{-1}^{*}+U_{0}^{*}+U_{1}^{*}}{3}=0 .
$$

Whereas for the local outputs,

$$
\begin{aligned}
& \mathrm{GU} \times u_{L}^{*}=\mathrm{GU} \times\left\{\begin{array}{l}
\frac{\left(r^{*} / R_{1}^{*}\right)\left(\left(U_{2}^{*}-U_{-1}^{*}\right) / 3\right)+\left(e^{*} /-E_{-1}^{*}\right)\left(\left(U_{1}^{*}-U_{-2}^{*}\right) / 3\right)}{1+2\left(e^{*} / E_{-1}^{*}\right)} \\
\text { for }\left(e^{*}, r^{*}\right) \in(-1,0) \text { th-block } \\
\frac{\left(r^{*} /-R_{-1}^{*}\right)\left(\left(U_{1}^{*}-U_{-2}^{*}\right) / 3\right)+\left(e^{*} / E_{1}^{*}\right)\left(\left(U_{2}^{*}-U_{-1}^{*}\right) / 3\right)}{1+2\left(r^{*} / R_{-1}^{*}\right)} \\
\text { for }\left(e^{*}, r^{*}\right) \in(0,-1) \text { th-block }
\end{array}\right. \\
& \approx \mathrm{GU} \times \mathrm{GR} \times\left\{\begin{array}{l}
\frac{\left(U_{2}^{*}-U_{-1}^{*}\right) / 3}{R_{1}^{*}}\left(r_{m}+\left(T / \frac{\mathrm{GR}}{\mathrm{GE}} \times \frac{-E_{-1}^{*}}{R_{1}^{*}} \times \frac{U_{2}^{*}-U_{-1}^{*}}{U_{1}^{*}-U_{-2}^{*}} T\right) e_{m}\right) \\
\frac{\left(U_{1}^{*}-U_{-2}^{*}\right) / 3}{-R_{-1}^{*}}\left(r_{m}+\left(T / \frac{\mathrm{GR}}{\mathrm{GE}} \times \frac{E_{1}^{*}}{-R_{-1}^{*}} \times \frac{U_{1}^{*}-U_{-2}^{*}}{U_{2}^{*}-U_{-1}^{*}} T\right) e_{m}\right) \\
\text { as }\left(e^{*}, r^{*}\right) \rightarrow\left(0^{+}, 0^{-}\right) .
\end{array}\right.
\end{aligned}
$$

According to the Lyapunov's indirect method [5] (Lyapunov's linearization method on stability [11]), a nonlinear controller and its linear counterpart(s) have the same local stability at the equilibrium point.

Note that the membership functions are symmetric around the equilibrium point for both SSMFs-FLC and ESMFs-FLC. Thus, the statements of local stability could be further simplified.

Property 14. For a given process, linear or nonlinear, and an SSMFs-FLC, the fuzzy feedback control system is asymptotically stable at the equilibrium point (set point) if and only if the linear PI control system with

$$
K_{p}=\mathrm{GU} \times \mathrm{GR} \times \frac{2 s_{u}^{2 m-2}+s_{u}^{2 m-1}}{6 s_{r}^{m-1}} \text { and } \tau_{I}=\frac{\mathrm{GR}}{\mathrm{GE}} \times\left(\frac{s_{e}}{s_{r}}\right)^{m-1} T
$$

is asymptotically stable. 
Property 15. For a given process, linear or nonlinear, and a ESMFS-FLC, the fuzzy feedback control system is asymptotically stable at the equilibrium point (set point) if and only if the linear PI control system with $K_{p}=\frac{1}{2}(\mathrm{GU} \times \mathrm{GR})$ and $\tau_{I}=(\mathrm{GR} / \mathrm{GE}) T$ is asymptotically stable.

\subsection{Global stability analysis for PD-type FLCs}

The USMFs-FLC could be functioned as a PD-type controller if its final crisp output is fed directly to the process for control. That is

$$
\mathrm{CO}(n)=u_{\mathrm{ss}}+\mathrm{GU} \times u^{*} \quad(\text { for PD-type FLC })
$$

where $u_{\mathrm{ss}}$ denotes initial steady controller output. It is known that a closed-loop system is $L_{p}$ stable if its two subsystems, the process and the controller, are both $L_{p}$ stable in themselves and if the "loop gain" is less than one for $1 \leqslant p \leqslant \infty$. Assume the process under consideration is $L_{\infty}$ stable. Then the subsequent work is to check whether the PD-type FLC is $L_{\infty}$ stable or not, i.e., to find if there exists an upper bound for the controller gain. Applying the small gain theory one can derive a stabilizing criterion for the nonlinear control system with the PD-type FLCs as controllers. The following properties elucidate the main results for stability of the PD-type fuzzy control system.

Property 16. The PD-type USMFs-FLC is $L_{\infty}$ stable, and its gain is always less than $K_{\mathrm{USMFs}-\mathrm{FLC}}^{\mathrm{PD}}$ where

$$
\frac{K_{\mathrm{USMFs}-\mathrm{FLC}}^{\mathrm{PD}}}{\mathrm{GU}} \equiv \max \left\{\begin{array}{c}
\max _{k \in I_{2 m}-\{-2 m, 2 m-1,2 m\}}\left[\mathrm{GE} \times \frac{\left(U_{k+2}^{*}-U_{k-1}^{*}\right) / 3}{E_{i+1}^{*}-E_{i}^{*}}+2 \mathrm{GR} \times \frac{\left(U_{k+3}^{*}-U_{k}^{*}\right) / 3}{R_{j+1}^{*}-R_{j}^{*}}\right] \\
\max _{k \in I_{2 m}-\{-2 m, 2 m-1,2 m\}}\left[\mathrm{GE} \times \frac{\left(U_{k+3}^{*}-U_{k}^{*}\right) / 3}{E_{i+1}^{*}-E_{i}^{*}}+2 \mathrm{GR} \times \frac{\left(U_{k+2}^{*}-U_{k-1}^{*}\right) / 3}{R_{j+1}^{*}-R_{j}^{*}}\right]
\end{array}\right\} .
$$

Proof. Consider output of the PD-type USMFs-FLC for regions I or IV on the $i j$ th-block,

$$
\begin{aligned}
u^{*} \leqslant & \frac{U_{k}^{*}+U_{k+1}^{*}+U_{k+2}^{*}}{3}+\left(\frac{r^{*}-R_{j}^{*}}{R_{j+1}^{*}-R_{j}^{*}}\right)\left(\frac{U_{k+3}^{*}-U_{k}^{*}}{3}\right)-\left(\frac{E_{i+1}^{*}-e^{*}}{E_{i+1}^{*}-E_{i}^{*}}\right)\left(\frac{U_{k+2}^{*}-U_{k-1}^{*}}{3}\right) \\
= & \left(\mathrm{GE} \times \frac{\left(U_{k+2}^{*}-U_{k-1}^{*}\right) / 3}{E_{i+1}^{*}-E_{i}^{*}}+\mathrm{GR} \times \frac{\left(U_{k+3}^{*}-U_{k}^{*}\right) / 3}{R_{j+1}^{*}-R_{j}^{*}}\right) e_{m}(n T)-\left(\mathrm{GR} \times \frac{\left(U_{k+3}^{*}-U_{k}^{*}\right) / 3}{R_{j+1}^{*}-R_{j}^{*}}\right) e_{m}(n T-T) \\
& +\underbrace{\frac{U_{k}^{*}+U_{k+1}^{*}+U_{k+2}^{*}}{3}-E_{i+1}^{*} \frac{\left(U_{k+2}^{*}-U_{k-1}^{*}\right) / 3}{E_{i+1}^{*}-E_{i}^{*}}-R_{j+1}^{*} \frac{\left(U_{k+3}^{*}-U_{k}^{*}\right) / 3}{R_{j+1}^{*}-R_{j}^{*}}}_{\equiv C_{\mathrm{I}, \mathrm{IV}}} \\
\Rightarrow & \sup \left|u^{*}\right| \leqslant \sup \left(\mathrm{GE} \times \frac{\left(U_{k+2}^{*}-U_{k-1}^{*}\right) / 3}{E_{i+1}^{*}-E_{i}^{*}}+2 \mathrm{GR} \times \frac{\left(U_{k+3}^{*}-U_{k}^{*}\right) / 3}{R_{j+1}^{*}-R_{j}^{*}}\right) \sup \left|e_{m}\right|+\sup \mid C_{\mathrm{I}, \mathrm{IV} \mid} \\
\equiv & K_{\mathrm{I}, \mathrm{IV} \sup \left|e_{m}\right|+\sup \mid C_{\mathrm{I}, \mathrm{IV} \mid}}
\end{aligned}
$$


Similarly, for input pattern on regions II or III of the $i j$ th-block,

$$
\begin{aligned}
\sup \left|u^{*}\right| \leqslant & \sup \left(\operatorname{GE} \times \frac{\left(U_{k+3}^{*}-U_{k}^{*}\right) / 3}{E_{i+1}^{*}-E_{i}^{*}}+2 \mathrm{GR} \times \frac{\left(U_{k+2}^{*}-U_{k-1}^{*}\right) / 3}{R_{j+1}^{*}-R_{j}^{*}}\right) \sup \left|e_{m}\right| \\
& +\sup \left|\frac{U_{k}^{*}+U_{k+1}^{*}+U_{k+2}^{*}}{3}-E_{i+1}^{*} \frac{\left(U_{k+3}^{*}-U_{k}^{*}\right) / 3}{E_{i+1}^{*}-E_{i}^{*}}-R_{j+1}^{*} \frac{\left(U_{k+2}^{*}-U_{k-1}^{*}\right) / 3}{R_{j+1}^{*}-R_{j}^{*}}\right| \\
\equiv & K_{\mathrm{II}, \text { III }} \sup \left|e_{m}\right|+\sup \left|C_{\mathrm{II}, \mathrm{III}}\right| .
\end{aligned}
$$

The property is proved by defining the maximum possible controller gain as

$$
\frac{K_{\mathrm{USMFs}}^{\mathrm{PDLC}}}{\mathrm{GU}}=\max \left\{K_{\mathrm{I}, \mathrm{IV}}, K_{\mathrm{II}, \mathrm{III}}\right\} .
$$

Property 17. The PD-type SSMFs-FLC (ESMFs-FLC) is $L_{\infty}$ stable, and its gain is always less than $K_{\mathrm{SSMFs}-\mathrm{FLC}}^{\mathrm{PD}}\left(K_{\mathrm{ESMFs}-\mathrm{FLC}}^{\mathrm{PD}}\right)$ where

$$
\begin{aligned}
& \frac{K_{\mathrm{SSMFs}-\mathrm{FLC}}^{\mathrm{PD}}}{\mathrm{GU}}=\left(\frac{\mathrm{GE}}{s_{e}^{m-1}}+\frac{2 \mathrm{GR}}{s_{r}^{m-1}}\right) \frac{2 m-(2 m-3) s_{u}^{3}}{6} \\
& \frac{K_{\mathrm{ESMFs}-\mathrm{FLC}}^{\mathrm{PD}}}{\mathrm{GU}}=\frac{1}{2}(\mathrm{GE}+2 \mathrm{GR}) .
\end{aligned}
$$

Proof. Note that

$$
\begin{aligned}
& \max _{\forall k \in I_{2 m}-\{2 m-1,2 m\}}\left\{\frac{U_{k+3}^{*}-U_{k}^{*}}{3}\right\}=\frac{U_{2 m}^{*}-U_{2 m-3}^{*}}{3}, \\
& \min _{\forall i \in I_{m}-\{m\}}\left\{E_{i+1}^{*}-E_{i}^{*}\right\}=E_{1}^{*}-E_{0}^{*} \text { and } \min _{\forall j \in I_{m}-\{m\}}\left\{R_{j+1}^{*}-R_{j}^{*}\right\}=R_{1}^{*}-R_{0}^{*} .
\end{aligned}
$$

Substituting these facts and definitions for related principal values for SSMFs and/or ESMFs into $K_{\text {USMFs-FLC }}$ leads to the property.

Property 18. A feedback control system with PD-type USMFs-FLC (SSMFs-FLC, ESMFs-FLC) as the controller is $L_{\infty}$ stable if the process gain, $\gamma_{\infty}$, is less than inverse of the maximum possible controller gain.

Proof. A straightforward application of the well-known small-gain theorem.

\section{Conclusion}

In this article, the authors analyze the mathematical input/output relation of an FLC equipped with a series of unequally spaced triangular fuzzy partitions as the membership functions (USMFs-FLC for short). Rigorous analyses prove that an USMFs-FLC, under the assumption of simple rule mapping, can be viewed as the combination of a global multilevel relay and a local nonlinear compensator. Such an analysis is a generalization to the similar works given by Ying [15] whose FLC is characterized by equally spaced membership functions. The influence of the distribution of principal values used to construct the unequally spaced membership functions of the FLC are also elucidated. The ultimate control behaviors as the number of 
linguistic terms for each input variable becomes infinity is illustrated. The local stability criteria for PI-type USMFs-FLCs are derived and the global stability properties for PD-type controllers are also established. Such theoretical analysis is significant for clarifying the inner characteristics of the FLCs. Obviously, the assumption

of simple control rules might limit the control performance and thus the controller needs further adjustment in practical applications. The feasible procedures for adaptive tuning of relevant design parameters and the pragmatic strategies for self-organization of control rules of a given FLC to enhance control performance are currently on-going projects.

\section{References}

[1] C.L. Chen, W.C. Chen, Fuzzy controller design by using neural network techniques, IEEE Fuzzy Systems 2 (1994) $235-244$.

[2] C.L. Chen, C.T. Hsieh, User-friendly design method for fuzzy logic controller, IEE Proc. Control Theory Appl. 143 (1996) $358-366$.

[3] C.L. Chen, F.C. Kuo, Design and analysis of a fuzzy logic controller, Int. J. Systems Sci. 26 (1995) 1223-1248.

[4] D. Driankov, H. Hellendoorn, M. Reinfrank, An Introduction to Fuzzy Control, Springer, Berlin, 1993.

[5] H. Khalil, Nonlinear Systems, Macmillan, New York, 1992.

[6] B. Kosko, Neural Networks and Fuzzy Systems, Prentice-Hall, Englewood Cliffs, NJ, 1992.

[7] C.C. Lee, Fuzzy logic in control systems: Fuzzy logic controller, part I, II, IEEE Trans. Systems Man Cybernet. 20 (1990) $404-435$.

[8] E.H. Mamdani, Applications of fuzzy algorithms for simple dynamic plant, IEE 121 (1974) 1585-1588.

[9] W. Qiao, M. Mizumoto, PID type fuzzy controller and parameters adaptive method, Fuzzy Sets and Systems 78 (1996) 23-35.

[10] W. Siler, H. Ying, Fuzzy control theory: The linear case, Fuzzy Sets and Systems 33 (1989) 275-290.

[11] J. Slotine, W. Li, Applied Nonlinear Control, Prentice-Hall, London, 1991.

[12] C. Wong, C. Chou, D. Mon, Studies on the output of fuzzy controller with multiple inputs, Fuzzy Sets and Systems 57 (1993) $149-158$.

[13] R.R. Yager, D.P. Filev, Essentials of Fuzzy Modeling and Control, Wiley, New York, 1994.

[14] O. Yagishita, O. Itoh, M. Sugeno, Application of fuzzy reasoning to the water purification process, in: M. Sugeno (Ed.), Industrial Applications of Fuzzy Control, Elsevier, New York, 1985, pp. 19-40.

[15] H. Ying, A nonlinear fuzzy controller with linear control rules is the sum of a global two-dimensional multilevel relay and a local nonlinear proportional-integral controller, Automatica 29 (1993) 499-505.

[16] H. Ying, General analytical structure of typical fuzzy controller and their limiting structure theorems, Automatica 29 (1993) $1139-1143$.

[17] H. Ying, The simplest fuzzy controllers using different inference methods are different nonlinear proportional-integral controllers with variable gains, Automatica 29 (1993) 1579-1589.

[18] H. Ying, Practical design of nonlinear fuzzy controllers with stability analysis for regulating processes with unknown mathematical models, Automatica 30 (1994) 1185-1195.

[19] H. Ying, W. Siler, J.J. Buckley, Fuzzy control theory: A nonlinear case, Automatica 26 (1990) 513-520. 